HD-THEP-01-36

\title{
Cosmon dark matter?
}

\author{
Christof Wetterich \\ Institut für Theoretische Physik, \\ Universität Heidelberg, \\ Philosophenweg 16, \\ D-69120 Heidelberg, Germany.
}

\begin{abstract}
We investigate if the fluctuations of the scalar field mediating quintessence the cosmon - can play an important role in cosmology. Small fluctuations with short wavelength behave similar to a relativistic gas. In contrast, the contribution to the energy density from horizon size fluctuations may decrease less rapidly than radiation. We discuss the possibility that the cosmon fluctuations grow nonlinearly, form lumps and constitute the clustering dark matter of the universe. Cosmon dark matter would lead to interesting consequences for the equation of state and the coupling between quintessence and dark matter.
\end{abstract}




\section{Introduction}

The idea of quintessence was born [1] from an attempt to understand the vanishing of the cosmological constant. In this work we proposed an attractor-type ("tracker") cosmological solution', where a scalar field, the cosmon, "rolls down" an exponential potential. This model predicts that part of the energy density of the universe occurs in the form of homogenous "dark energy" as a dynamical quantity evolving in time [1]-[0]. In contrast to the necessity of an extreme fine tuning for a nonzero cosmological constant, the cosmon model implies that today's presence of the dark energy component would be independent of details of the model and the initial conditions. It was realized [1] that the late universe could become cosmon-dominated and that mild deviations of the potential from an exact exponential form could affect the precise present cosmological behavior. Modifications of the age of the universe as compared to standard Friedman cosmology depend on the precise form of the potential and the coupling of the cosmon to dark matter [1], [4], [6]. Various shapes fo the potential have been investigated subsequently, like the power law potential proposed by Ratra and Peebles [2].

Observable cosmology, and in particular the possibility that the universe accelerates today, depend crucially on the present effective equation of state of quintessence [5], [7]. The fraction of homogenous scalar dark energy within the total energy density, $\Omega_{h}$, should have been small during structure formation [8]. On the other hand, today's value of $\Omega_{h}$ may be as large as 0.6-0.7 if one compares the inhomogenous "clumping" part of dark matter $\Omega_{\text {inhom }} \lesssim 0.4$ and the verified [9] prediction of inflation $\Omega_{t o t}=1$. Combining these requirements one concludes that $\Omega_{h}$ must have grown substantially after the end of structure formation. This is realized by a negative pressure of quintessence [7] and leads to a presently accelerating universe as suggested by recent supernovae 1a observations [10].

Present quintessence models which lead to a realistic cosmological phenomenology (at least according to what is believed today) seem to be somewhat in conflict with the original motivation of quintessence, namely that today's fraction of the dark energy, $\Omega_{h}\left(t_{0}\right)$, should not depend sensitively on the model or the initial conditions. The power-law potentials require some tiny parameter or ratio of mass scales which is chosen such that $\Omega_{h}$ plays a role just today. Potentials with a minimum [12], [13] need a finetuning of its height, location and the curvature around it. Even the perhaps more natural modifications of the exponential potential [14 need a tuning of parameters at the percent level which singles out indirectly "today" as a special moment in cosmological history.

Besides the problem of understanding the origin of quintessence from a unified theory and its relation to the cosmological constant, we see two major open issues in the quintessence scenario:

\footnotetext{
${ }^{1}$ For more details see [2], [3], [4].

${ }^{2}$ Recent bounds on the present and past properties of quintessence can be found in [1].
} 
(1) If homogenous quintessence accounts for 60-70\% of the total energy density today, what is the nature of the remaining 30-40 \% of inhomogenous dark matter? Could it be the inhomogenous fluctuations of the cosmon field? In this case the cosmon would account for all dark matter (except perhaps a small fraction in neutrinos). The space average of the cosmon field would be responsible for the homogenous dark energy, whereas its local fluctuations around the average value could give rise to the inhomogenous part of dark matter which forms structure. We may call such a form of inhomogenous dark matter "cosmon dark matter". Cosmon dark matter could give a natural explanation why it is of similar importance as homogenous quintessence.

(2) What has triggered the recent change of the properties of quintessence (equation of state) or its role in cosmology such that the universe accelerates at present? Why does acceleration happen just now?

The two questions may not be unrelated. Both may have to do with the importance of nonlinearities for the cosmon fluctuations. Obviously, a comparable amount of cosmon dark matter and homogenous quintessence requires that the cosmon fluctuations are not small at present. Let us next ask what special event in the present cosmological epoch could have modified the effective cosmological evolution equations. A possible answer is that structure formation has essentially taken place [14]. The existence and distribution of galaxies and clusters corresponds to strong nonlinearities in the fluctuations of inhomogenous dark matter. The two-point correlation function is large as compared to the squared average energy density. Also the higher correlation functions for the fluctuations in the inhomogenous energy density are comparable to the two-point function, in contrast to early cosmology. Can these nonlinearities trigger [15] the increase of $\Omega_{h}$ and the acceleration of the universe by an effect of "backreaction"? Or can the equation of state of cosmon dark matter be negative by itself? An accelerating epoch would then necessarily begin only after structure formation.

We will see that small cosmon fluctuations on scales much smaller than the horizon behave essentially as relativistic matter [2], [8], [16]. Small-scale linear fluctuations cannot grow and can therefore not account for structure formation. Since in the present epoch characteristic galactic scales are much smaller than the horizon, one concludes that the cosmon dark matter scenario can only be realized if the nonlinearities in the cosmon fluctuations play an important role. In this context it should be noted that the average energy density arising from the local cosmon field fluctuations may be distributed much more smoothly than the individual cosmon fluctuations during a certain period of the cosmological evolution. (This is partly similar to the local fluctuations in the electromagnetic field which may be substantial - corresponding to the photons of a relativistic gas - and nevertheless account for a rather homogenous energy density in radiation.) The compatibility of an approximately homogenous cosmon energy density (related to cosmon fluctuations and not to be confounded with homogenous quintessence) with large average inhogeneities in the scalar field opens two interesting alternatives. Either the cosmon 
fluctuations were never linear and only the energy density was smooth in early cosmology. Or the scalar fluctuations started in the linear regime and have grown large in the course of the cosmological evolution.

In this paper we concentrate on the second alternative which is easier accessible for quantitative studies with existing methods. Nevertheless, our results will also shed some light on the first alternative. One may imagine the following cosmological scenario: (1) In an early radiation-dominated epoch of cosmology the cosmon fluctuations and the energy density in cosmon dark matter are small. The homogenous quintessence would adjust to the dominant radiation according to an attractor solution. This can lead to a small nonvanishing $\Omega_{h}(t) \lesssim 0.1$ during this period, compatible with nucleosynthesis. (2) For an effective equation of state for cosmon dark matter similar to nonrelativistic matter, or between nonrelativistic and relativistic matter, the part of cosmon dark matter $\Omega_{c}$ would grow relative to the radiation. Cosmon dark matter could overwhelm the radiation at a certain moment in the history of the universe. A cosmon-dominated epoch begins. (3) Subsequently, structure formation could take place by growth of the inhomogeneities of the energy density in cosmon dark matter. (4) Once structure has formed, the higher correlation functions for the energy density of the cosmon fluctuations have also grown large. Their backreaction could modify the effective dynamical equation for gravity and the average cosmon field and trigger the increase of $\Omega_{h}$. In particular, the coupling of homogenous quintessence to dark matter may become important [1], [17]. (5) The universe ends in a quintessence-dominated state. We are aware that these ideas are still speculative. We will sketch in the following only the general setting which could describe the cosmon dark matter scenario. Obviously, alternative solutions to the two open issues mentioned before can be envisaged within quintessence [18.

In sect. 2 we write down the field equations which couple the time evolution of the homogenous "average fields" to the fluctuations. Sect. 3 discusses the simple case of small cosmon fluctuations inside the horizon. They behave similar to a relativistic gas. Nevertheless, we argue in sect. 4 that the fluctuation spectrum of the cosmon needs not to be dominated by the short wavelength fluctuations. We advocate a natural scenario where the energy density of the cosmon fluctuations is dominated by wavelengths of the order of the horizon - at least as long as they remain linear. The maximum of the spectrum becomes therefore time-dependent.

Cosmon fluctuations with wave length around or larger than the horizon need the inclusion of the gravity fluctuations. We discuss the coupled linear system of fluctuation equations in sect. 5. We adopt for this purpose the harmonic background gauge which we believe gives a simple picture of the dominant physical processes - in contrast to the synchronous gauge where the growing modes for large wavelength do not correspond to growing gauge-invariant quantities. The growth of the cosmon fluctuations depends on the homogenous "background" solution. In sect. 6 we present several scenarios for background solutions for the specific model of quintessence with an exponential potential. 
For this class of models we compute in sect. 7 the growth exponent for the large scale cosmon fluctuations.

In sect. 8 we turn to the radiation-dominated epoch. We establish that the very large-scale cosmon fluctuations remain constant for a large class of exponential cosmon potentials. However, it seems not unnatural that the horizon size fluctuations could grow. Whereas the contribution to the energy density from very short and very long wavelength fluctuations decreases $\sim t^{-2}$ for the case of constant large-scale fluctuations, the energy density of the "horizon component" of the cosmon fluctuations typically could decrease somewhat slower. As a result, the horizon component of the cosmon fluctuations would become more important than radiation at a certain moment in the history of the universe, such that the radiation epoch ends and cosmology transites to a period where cosmon dark matter dominates. This would be a scenario where cosmon fluctuations could grow nonlinear and form "cosmon lumps" - these may even be possible candidates for (some) galaxies [19]. We address general aspects of a cosmon-dominated universe only shortly in sect. 9 and end our discussion with a first account of the role of the nonlinearities for the effective cosmon fluctuation equations in sect. 10. A mean field computation shows the tendency that non-relativistic fluctuation behavior may shift to wavelengths further inside the horizon. Our conclusions are presented in sect. 11. Two appendices A, B give details for the fluctuation equation for gravity and for the gravitational contribution to the energy momentum tensor. For the convenience of the reader we summarize our naming scheme for the various contributions to the energy density in appendix $\mathrm{C}$.

\section{Cosmological equations for fluctuating fields}

We consider first only a scalar field $\varphi$ coupled to gravity and omit radiation. This may be a reasonable approximation for late cosmology where photons, leptons, and baryons can be treated as a small correction. For early cosmology radiation will be added later. We will work within a formalism that is equally suited for linear and nonlinear cosmon fluctuations. Let us start with the Einstein- and Klein-Gordon equations in a statistical ensemble

$$
\begin{gathered}
<R_{\mu \nu}-\frac{1}{2} R g_{\mu \nu}>=\frac{1}{2 M^{2}}\left\{-<V g_{\mu \nu}>+<\partial_{\mu} \chi \partial_{\nu} \chi>-\frac{1}{2}<\partial^{\rho} \chi \partial_{\rho} \chi g_{\mu \nu}>\right\} \\
<\chi ;_{\mu}^{\mu}>=<V^{\prime}>
\end{gathered}
$$

where the brackets stand for the ensemble average. Here $V$ denotes the scalar potential and $V^{\prime}=\partial V / \partial \chi$. These field equations can be derivedf from a "microscopic action"

\footnotetext{
${ }^{3}$ We use a metric signature $(-,+,+,+)$ and $R_{\mu \nu \rho}{ }^{\lambda}=-\partial_{\mu} \Gamma_{\nu \rho}{ }^{\lambda}+\ldots$

${ }^{4}$ Conceptually, the microscopic action is an effective action of a quantum field theory where quantum fluctuations are already included. A local patch of the universe obeys the "microscopic field equations"
} 


$$
\left(M^{2}=M_{p}^{2} / 16 \pi\right) \quad S=\int d^{4} x \sqrt{g}\left\{-M^{2} R+V(\chi)+\frac{1}{2} \partial^{\mu} \chi \partial_{\mu} \chi\right\}
$$

The statistical averages denoted by $<>$ depend, in principle, on the particular observation and on the properties of the observable local piece of the universe. As a typical size for our "local piece" we may choose a redshift $z \leq 10$. We will assume here that our local region of the universe reflects the average properties of a much larger region of the universe (typically extending far beyond our horizon). In this case the statistical ensemble should be taken as homogenous and isotropic.

For a homogenous and isotropic ensemble we expand around a spatially flat "background" Robertson-Walker metric $\bar{g}_{\mu \nu}(t)=<g_{\mu \nu}>=\operatorname{diag}\left(-1, a^{2}(t), a^{2}(t), a^{2}(t)\right.$ ) (with scale factor $a(t)$ and $H(t)=\dot{a}(t) / a(t))$ and a background scalar field $\varphi(t)=<\chi>$, i.e.

$$
\begin{aligned}
g_{\mu \nu} & =\bar{g}_{\mu \nu}+h_{\mu \nu} \\
\chi & =\varphi+\delta \chi \\
<h_{\mu \nu}> & =0, \quad<\delta \chi>=0
\end{aligned}
$$

Insertion into eqs. (2.1), (2.2) yields the field equations

$$
\begin{gathered}
\bar{R}_{\mu \nu}-\frac{1}{2} \bar{R} \bar{g}_{\mu \nu}=\frac{1}{2 M^{2}}\left\{-V(\varphi) \bar{g}_{\mu \nu}+\partial_{\mu} \varphi \partial_{\nu} \varphi-\frac{1}{2} \partial^{\rho} \varphi \partial_{\rho} \varphi \bar{g}_{\mu \nu}+T_{\mu \nu}\right\} \\
-\varphi ;_{\mu}^{\mu}+V^{\prime}(\varphi)=q_{\varphi}
\end{gathered}
$$

with energy momentum tensor

$$
\begin{aligned}
T_{00} & =\rho=\rho_{c}+\rho_{g} \\
T_{i j} & =p \bar{g}_{i j}=\left(p_{c}+p_{g}\right) \bar{g}_{i j}
\end{aligned}
$$

The energy momentum tensor and the quantity $q_{\varphi}$ reflect the fluctuations $(i=1 . .3)$. Here the scalar fluctuations lead to

$$
\begin{aligned}
\rho_{c}(t)= & -<V(\varphi+\delta \chi) g_{00}>-V(\varphi)+<\dot{\chi}^{2}> \\
& -\frac{1}{2}<\partial^{\rho} \chi \partial_{\rho} \chi g_{00}>-\frac{1}{2} \dot{\varphi}^{2} \\
p_{c}(t)= & -\frac{1}{3 a^{2}(t)}<V(\varphi+\delta \chi) g_{i i}>+V(\varphi)+\frac{1}{3 a^{2}(t)}<\partial_{i} \chi \partial_{i} \chi> \\
& -\frac{1}{6 a^{2}(t)}<\partial^{\rho} \chi \partial_{\rho} \chi g_{i i}>-\frac{1}{2} \dot{\varphi}^{2} \\
q_{\varphi}(t)= & -<V^{\prime}(\varphi+\delta \chi)>+V^{\prime}(\varphi)+<\chi_{;_{\mu}}^{\mu}>-\varphi_{;_{\mu}}^{\mu}
\end{aligned}
$$

which are derived by variation of eq. (2.3) and correspond to eqs. (2.1), (2.2) without the brackets. The statistical mean averages over many such patches. The ensemble therefore consists of realizations obeying each the microscopic field equations.

${ }^{5}$ The incoherent contribution $q_{\varphi}$ to the scalar field equation was pointed out in ref. [6]. 
whereas the pure metric fluctuations induce

$$
\begin{aligned}
& \rho_{g}(t)=-2 M^{2}\left(<R_{00}-\frac{1}{2} R g_{00}>-\bar{R}_{00}+\frac{1}{2} \bar{R} \bar{g}_{00}\right) \\
& p_{g}(t)=-\frac{2 M^{2}}{3 a^{2}(t)}\left(<R_{i i}-\frac{1}{2} R g_{i i}>-\bar{R}_{i i}+\frac{1}{2} \bar{R} \bar{g}_{i i}\right)
\end{aligned}
$$

The Bianchi identity for $\bar{R}_{\mu \nu}$ implies the relation [1] [6]

$$
\dot{\rho}+3 H(\rho+p)+q_{\varphi} \dot{\varphi}=0
$$

This equation, together with the $0-0$-components of eq. (2.5) and eq. (2.6),

$$
\begin{gathered}
H^{2}=\frac{1}{6 M^{2}}\left(\rho+V(\varphi)+\frac{1}{2} \dot{\varphi}^{2}\right) \\
\ddot{\varphi}+3 H \dot{\varphi}+V^{\prime}(\varphi)=q_{\varphi}
\end{gathered}
$$

specifies the evolution of the background fields. The quantities $\rho, p$ and $q_{\varphi}$ express the "backreaction" of the fluctuations on the effective field equations for the mean fields ${ }^{6}$.

The system of equations (2.10)-(2.12) can be solved if an "equation of state" $p(\rho)$ and similarly the functional dependence $q_{\varphi}(\rho)$ are known. In general, the effective functions $p(\rho)$ and $q_{\varphi}(\rho)$ may be quite complicated and depend on the precise "initial conditions" for the fluctuations and on time. We assume here that the system has "locally equilibrated" with an effective equation of state, similar to hydrodynamcis. Before addressing the issue of large nonlinear scalar fluctuations it seems useful to understand the behavior of small fluctuations.

\section{Small cosmon fluctuations inside the horizon}

For small fluctuations one can expand around the homogenous field in second order in the fluctuations. For a first investigation we also neglect the mixing of the scalar fluctuations with the metric fluctuations. We will see below (sect. 5) that this is justified only if $\rho_{c}, p_{c}, q_{\varphi}$ are dominated by cosmon fluctuations well inside the horizon. Nevertheless, the approximation of inserting the background metric in eq. (2.8) shows already several important features in the most simple language. The fluctuations of the metric will be included in sect. 5. Inserting the quadratic approximations for $V(\varphi+\delta \chi)$ and $V^{\prime}(\varphi+\delta \chi)$ one finds

$$
\rho_{c}=\frac{1}{2} V^{\prime \prime}(\varphi) A+\frac{1}{2} B+\frac{C}{2 a^{2}}
$$

\footnotetext{
${ }^{6}$ See [20] for other effects of backreaction on quintessence.
} 


$$
\begin{aligned}
& p_{c}=-\frac{1}{2} V^{\prime \prime}(\varphi) A+\frac{1}{2} B-\frac{C}{6 a^{2}} \\
& q_{\varphi}=-\frac{1}{2} V^{(3)}(\varphi) A .
\end{aligned}
$$

In terms of the Fourier modes $\delta \chi_{k}\left(\delta \chi(x)=\int \frac{d^{3} k}{(2 \pi)^{3}} e^{i \vec{k} \vec{x}} \delta \chi_{k}\right.$ and $\vec{x}$ are comoving coordinates) the quantities $A, B, C$ are defined as

$$
\begin{aligned}
A & =<\delta \chi^{2}(x)>=\int \frac{d^{3} k}{(2 \pi)^{3}} \alpha_{k} \\
B & =<\delta \dot{\chi}^{2}(x)>=\int \frac{d^{3} k}{(2 \pi)^{3}} \beta_{k} \\
C & =<\partial_{i} \chi(x) \partial_{i} \chi(x)>=\int \frac{d^{3} k}{(2 \pi)^{3}} k^{2} \alpha_{k}
\end{aligned}
$$

with

$$
<\delta \chi_{k}^{*} \delta \chi_{k^{\prime}}>=(2 \pi)^{3} \delta\left(k-k^{\prime}\right) \alpha_{k}, \quad<\delta \dot{\chi}_{k}^{*} \delta \dot{\chi}_{k^{\prime}}>=(2 \pi)^{3} \delta\left(k-k^{\prime}\right) \beta_{k}
$$

Our aim is an understanding of the size and time evolution of $A, B$ and $C$ and thereby of the equation of state for cosmon dark matter. For small fluctuations the basic ingredient is the linearized microscopic field equation for the scalar fluctuations (for $k \neq 0)$ 】

$$
\delta \ddot{\chi}_{k}+3 H \delta \dot{\chi}_{k}+\frac{k^{2}}{a^{2}} \delta \chi_{k}+V^{\prime \prime} \delta \chi_{k}=0 .
$$

This can be used in order to derive an evolution equation for $A$

$$
\ddot{A}+3 H \dot{A}+4 V^{\prime \prime}(\varphi) A=-2\left(\rho_{c}-3 p_{c}\right)
$$

For a given equation of state $p_{c}\left(\rho_{c}\right)$ its solution determines $q_{\varphi}$. For attractor solutions with an exponential cosmon potential one has $V^{\prime \prime}=(9 / 2) H^{2}$ and the effective cosmon mass is proportional to the Hubble parameter [1], [1]. We will, however, keep our discussion general and consider arbitrary $V^{\prime \prime}(\varphi(t))$.

In the linear approximation there is no scattering, and the different $k$-modes evolve independently. We may define a mode number density $n_{k}$ as

$$
n_{k}=\frac{1}{2}\left(\frac{k^{2}}{a^{2}}+V^{\prime \prime}\right)^{-1 / 2}\left\{\left(\frac{k^{2}}{a^{2}}+V^{\prime \prime}\right) \alpha_{k}+\beta_{k}\right\}
$$

In terms of $n_{k}$ and the quantity $\Delta_{k}$

$$
\Delta_{k}=\frac{1}{2}\left(\frac{k^{2}}{a^{2}}+V^{\prime \prime}\right)^{-1 / 2}\left\{\left(\frac{k^{2}}{a^{2}}+V^{\prime \prime}\right) \alpha_{k}-\beta_{k}\right\}
$$

\footnotetext{
${ }^{7}$ Note that by assumption $\left(q_{\varphi}\right)_{k \neq 0}=0$ and $\chi_{k \neq 0}=\delta \chi_{k \neq 0}$. The equation for fluctuations with $k=0$ differs from eq. (3.4) if $q_{\varphi} \neq 0$.
} 
we can write

$$
\rho_{c}=\int \frac{d^{3} k}{(2 \pi)^{3}} \rho_{k}, p_{c}=\int \frac{d^{3} k}{(2 \pi)^{3}} p_{k}
$$

with

$$
\begin{aligned}
\rho_{k} & =\left(\frac{k^{2}}{a^{2}}+V^{\prime \prime}\right)^{1 / 2} n_{k}, \\
p_{k} & =\frac{k^{2}}{3 a^{2}}\left(\frac{k^{2}}{a^{2}}+V^{\prime \prime}\right)^{-1 / 2} n_{k}-\left(V^{\prime \prime}+\frac{2 k^{2}}{3 a^{2}}\right)\left(\frac{k^{2}}{a^{2}}+V^{\prime \prime}\right)^{-1 / 2} \Delta_{k}
\end{aligned}
$$

For $\Delta_{k}=0$ eq. (2.8) reduces to the standard relavistic relation for a collection of point particles with mass $\sqrt{\left|V^{\prime \prime}\right|}$ and momentum $\vec{k} / a$. Furthermore, for $\Delta_{k}=0$ the particle number is conserved for each $k$-mode

$$
\begin{aligned}
\frac{d}{d t}\left(n_{k} a^{3}\right) & =(3 H+\sigma) \Delta_{k} a^{3} \\
\sigma & =\frac{d}{d t} \ln \sqrt{\frac{k^{2}}{a^{2}}+V^{\prime \prime}}
\end{aligned}
$$

Possible unusual features are therefore all related to the "particle production $\left[\right.$ rate" $\sim \Delta_{k}$. The latter obeys the evolution equation

$$
\frac{d}{d t}\left(\Delta_{k} a^{3}\right)=(3 H+\sigma) n_{k} a^{3}+2\left(\frac{k^{2}}{a^{2}}+V^{\prime \prime}\right)^{1 / 2} \gamma_{k} a^{3}
$$

where $\gamma_{k}$ is defined by

$$
\frac{1}{2}<\delta \dot{\chi}_{k}^{*} \delta \chi_{k^{\prime}}+\delta \chi_{k}^{*} \delta \dot{\chi}_{k^{\prime}}>=(2 \pi)^{3} \delta\left(k-k^{\prime}\right) \gamma_{k}
$$

In particular, we note that in the absence of sizeable particle production the spectrum of $n_{k}$ has a time-invariant shape. Each modes scales separately according to $n_{k}(t) \sim$ $a^{-3}(t) n_{k}\left(t_{0}\right)$.

Consider first the contribution of modes with physical wave length well within the horizon, i.e. $k^{2} / a^{2} \gg H^{2}$, or a situation where $V^{\prime \prime} \gg H^{2}$. For $\frac{k^{2}}{a^{2}}+V^{\prime \prime} \gg H^{2}$ the modes perform damped oscillations. The oscillation period will be much shorter than $H^{-1}$ or the rate of change of $V^{\prime \prime}$. In leading order one may therefore neglect the damping term $\sim 3 H \delta \dot{\chi}$, the change in $a$ and the time variation of $V^{\prime \prime}$ in eq. (3.4). One infers the frequencies $\omega_{k}^{2}=k^{2} / a^{2}+V^{\prime \prime}$. After averaging over initial conditions typical for the ensemble, this leads to

$$
\beta_{k}=\left(\frac{k^{2}}{a^{2}}+V^{\prime \prime}\right) \alpha_{k}, \quad \Delta_{k}=0
$$

\footnotetext{
8 "Mode number production" would perhaps be a more approriate naming.
} 
The damping corrections to eq. (3.13) are suppressed by powers of $H^{2}\left(\frac{k^{2}}{a^{2}}+V^{\prime \prime}\right)^{-1}$. This also holds for corrections from the time evolution of $V^{\prime \prime}$. In particular, for modes with $k^{2} / a^{2}$ much larger than $V^{\prime \prime}$ and $H^{2}$ the corrections decrease with time $\sim H^{2} a^{2}$. (If the energy density has once be dominated by such "oscillating modes", this will remain so later.)

For $\Delta_{k}=0$ the mode number density $n_{k}$ is directly related to $\alpha_{k}$

$$
n_{k}=\left(\frac{k^{2}}{a^{2}}+V^{\prime \prime}\right)^{1 / 2} \alpha_{k}
$$

and one infers

$$
\rho_{k}=\left(\frac{k^{2}}{a^{2}}+V^{\prime \prime}\right) \alpha_{k} \quad, \quad p_{k}=\frac{k^{2}}{3 a^{2}} \alpha_{k}
$$

This implies the equation of state

$$
\begin{aligned}
& p / \rho=\frac{1}{3}\left(1+\frac{a^{2} V^{\prime \prime} A}{C}\right)^{-1} \\
& q_{\varphi}=-\frac{V^{(3)}(\varphi)}{2 V^{\prime \prime}(\varphi)}(\rho-3 p)
\end{aligned}
$$

We note that for an exponential potential $V=V_{0} \exp (-\alpha \varphi / M)$ and for a constant equation of state

$$
p=\frac{n-3}{3} \rho
$$

one can write [4]

$$
q_{\varphi}=\frac{\beta}{M} \rho
$$

with

$$
\beta=\frac{\alpha}{2}(4-n)
$$

(Cosmologies with $\beta \neq 0$ have been discussed extensively in [4, 17]).

We observe that for $V^{\prime \prime} \ll C /\left(a^{2} A\right)$ the scalar fluctuations behave like relativistic matter $(p \approx \rho / 3)$ with $q_{\varphi} \approx 0$. On the other hand, if the mass term dominates, $V^{\prime \prime} \gg C /\left(a^{2} A\right)$, one finds nonrelativistic behavior $p \approx 0$ with $q_{\varphi} \approx-V^{(3)} \rho /\left(2 V^{\prime \prime}\right)$. We typically concentrate on cosmon potentials where $V^{\prime \prime}$ decreases with time faster than $a^{-2}$. (In particular, for $V^{\prime \prime} \sim t^{-2}$ and a radiation-dominated universe $\left(a \sim t^{1 / 2}\right)$ or matter-dominated universe $\left(a \sim t^{2 / 3}\right)$ one has $V^{\prime \prime} a^{2} \sim t^{-1}$ or $t^{-2 / 3}$, respectively. $)$ Then an individual mode with given wavelength $\sim k^{-1}$ has the tendency to end in the relativistic regime for large enough $t$.

In order to proceed further with a computation of the cosmon equation of state, we need information about the initial fluctuation spectrum and its time evolution. As an illustration let us first briefly consider fluctuation spectra for which $A / C$ remains 
essentially constant or varies slowly as compared to $V^{\prime \prime} a^{2}$. In this case the equation of state for cosmon dark matter approaches a relativistic gas for large $t$. Indeed, the relativistic modes $\left(\frac{k^{2}}{a^{2}} \gg V^{\prime \prime}\right)$ remain relativistic in the course of the evolution, and nonrelativistic modes $\left(\frac{k^{2}}{a^{2}} \ll V^{\prime \prime}\right)$ become relativistic at a later stage. If the cosmon "energy spectrum" $k^{3} \rho_{k}$ would be peaked around a fixed comoving wave number $\bar{k} \neq 0$ (as for the microwave background radiation), the cosmon dark matter would behave like relativistic matter after some time $t_{r}$, where $\bar{k}^{2} / a^{2}\left(t_{r}\right) \approx V^{\prime \prime}\left(t_{r}\right)$. If, furthermore, $t_{r}$ is in our past, such cosmon dark matter could not be relevant today. For typical quintessence models the squared mass $V^{\prime \prime}$ is of the order of $H^{2}$. Then "linear cosmon dark matter" (small fluctuations) behave like relativistic matter if they are dominated by fluctuations with wavelength well within the horizon. In this case the cosmon dark matter may constitute a certain fraction of the radiation [16]. This gives a stationary contribution to $\Omega$ during the radiation-dominated epoch. Just as for the rest of the radiation this component will become insignificant during a "matter-dominated period". In particular, it cannot play the role of dark matter and cannot be responsible for the growth of structure.

In the following we will turn to the more interesting scenario where the cosmon fluctuations with wavelength around and larger than the horizon play an important role. Indeed, since the cosmons interact only gravitationally with ordinary matter, it seems unlikely that the cosmon fluctuations are produced thermally. There is therefore no reason for a peak in $k^{3} \rho_{k}$ at some fixed $\bar{k} \neq 0$ (as for thermally produced relics). We rather suppose that the cosmon fluctuations have been produced out of equilibrium at a very early stage of the cosmological evolution, typically during inflation.

For the modes with $k^{2} / a^{2} \ll V^{\prime \prime}$, the neglection of gravity is actually not a valid approximation. For an appreciation of the gravitational effects discussed in sect. 5 we will nevertheless briefly mention here the consequences of their omission. With $H=\eta t^{-1}$, $V^{\prime \prime}+\frac{k^{2}}{a^{2}}=\zeta_{k} t^{-2}$ and $\tau=\ln \left(t / t_{0}\right)$ the long-distance modes obey the field equation

$$
\frac{d^{2}}{d \tau^{2}} \delta \chi_{k}+(3 \eta-1) \frac{d}{d \tau} \delta \chi_{k}+\zeta_{k} \delta \chi_{k}=0
$$

If the $\tau$-dependence of $\eta$ and $\zeta_{k}$ can be neglected (i.e. $V^{\prime \prime}$ dominates $\zeta_{k}$ ), the solution reads (with $\omega_{k}, \gamma_{k}$ real)

$$
\delta \chi_{k}(\tau)=e^{i \omega_{k} \tau} e^{-\gamma_{k} \tau} \delta \chi_{k}(0)
$$

with

$$
\gamma=\frac{3 \eta-1}{2} \quad, \quad \omega_{k}^{2}=\zeta_{k}-\frac{(3 \eta-1)^{2}}{4}
$$

This leads to

$$
\beta_{k}=t^{-2}\left(\omega_{k}^{2}+\gamma^{2}\right) \alpha_{k}=\left(\frac{k^{2}}{a^{2}}+V^{\prime \prime}\right) \alpha_{k}
$$

We conclude that without gravity effects the "particle production" $\Delta_{k}$ is negligible both for modes with $k^{2} / a^{2}+V^{\prime \prime} \gg H^{2}$ and $\frac{k^{2}}{a^{2}} \ll V^{\prime \prime}$. We will see below, however, that $\Delta_{k}$ 
is actually of the same size as $n_{k}$ once gravity effects are included for the long-distance modes.

\section{Cosmon spectrum}

The equation of state of cosmon dark matter depends strongly on the spectrum of the cosmon fluctuations. In contrast to particles that have once been in thermal equilibrium the cosmon fluctuations are typically produced by out of equilibrium physics in very early cosmology (e.g. inflation). We have little a priori knowledge about the primordial spectrum without understanding the role of the cosmon in this very early epoch. Nevertheless, the spectrum is also reshaped ("processed") by the different time evolution for modes with different $k$. As a result, we will see that a spectrum with a maximum in the vicinity of the momentum corresponding to the horizon appears to be rather natural.

Let us parametrize our lack of knowledge by a general form of the spectrum

$$
\alpha_{k}(t)=\bar{\alpha}(t) M^{2} k^{\nu_{I R}-3}\left(1+k / k_{p}(t)\right)^{\nu_{U V}-\nu_{I R}}
$$

For small and large $k$, it has the properties

$$
k^{3} \alpha_{k} \sim \begin{cases}k^{\nu_{I R}} & \text { for } k \ll k_{p} \\ k^{\nu_{U V}} & \text { for } k \gg k_{p}\end{cases}
$$

This spectrum leads to a finite energy density if the power laws for small and large $k$ obey

$$
\nu_{I R}>0 \quad, \quad \nu_{U V}<-2
$$

It is peaked around a characteristic comoving momentum $k_{p}(t)$ which may depend on time. Performing the integrals (3.2) one finds

$$
A=\mathcal{A}(t) M^{2} k_{p}(t)^{\nu_{I R}} \quad, \quad C=c_{p} k_{p}^{2}(t) A
$$

The generic form of eq. (4.4) holds for a wide class of spectra with scaling properties similar to eq. (4.1), whereas the constant $c_{p}$ depends on the particular shape and reads in our case

$$
c_{p}\left(\nu_{I R}, \nu_{U V}\right)=\frac{\Gamma\left(\nu_{I R}+2\right) \Gamma\left(-\nu_{U V}-2\right)}{\Gamma\left(\nu_{I R}\right) \Gamma\left(-\nu_{U V}\right)}
$$

We may distinguish three types of qualitatively different behavior:

i) horizon-dominated spectrum: in this case the physical momentum corresponding to the peak of the spectrum scales with the horizon, i.e.

$$
k_{p}(t)=\kappa H(t) a(t)
$$


ii) radiation-like spectrum: here $k_{p}(t)$ is a fixed comoving wave number with corresponding physical length scale well within the horizon, such that $k_{p} / a(t) \gg H(t)$.

iii) infrared spectrum: it is dominated by wave lengths outside the horizon, with $k_{p} / a(t) \ll H(t)$.

The normalization $\mathcal{A}(t)$ is proportional to $\bar{\alpha}(t)$. It is treated here as a free parameter. We remind, however, that the cosmon fluctuations have a direct impact on the fluctuations in the cosmic microwave background and are therefore restricted in amplitude at the time of last scattering. In fact, the microscopic fluctuations of the cosmon dark energy $\delta \rho_{k}$ gets a contribution that is linear in the fluctuations $\delta \chi_{k}, \delta \dot{\chi}_{k}$. This is related to the fact that the cosmon does not oscillate around a constant value, in contrast to photons and baryons. For example, the contribution to the energy density $\sim V^{\prime} \delta \chi$ does not vanish since the background field is not at a minimum of $V$ and therefore $V^{\prime} \neq 0$. As a consequence, the metric fluctuations $\left(h_{\mu \nu}\right)_{k}$ and therefore also the temperature fluctuations $(\delta T / T)_{k}$ in the cosmic microwave spectrum get a contribution $\sim \alpha_{k}, \beta_{k}$. It is conceivable, nevertheless, that the proportionality constant is rather small such that rather large cosmon fluctuations induce only small fluctuations in the radiation.

For the spectrum (4.1) we can determine the evolution of $\mathcal{A}(t)$ from the evolution of the modes with momenta smaller than $k_{p}$

$$
\partial \ln \mathcal{A}(t) / \partial \ln t=\partial \ln \alpha_{k \ll k_{p}}(t) / \partial \ln t=f_{<}
$$

On the other hand, the combination $\mathcal{A} k_{p}^{\nu_{I R}-\nu_{U V}}$ scales with the modes $k \gg k_{p}$

$$
\partial \ln \mathcal{A}(t) / \partial \ln t+\left(\nu_{I R}-\nu_{U V}\right) \partial \ln k_{p}(t) / \partial \ln t=\partial \ln \alpha_{k \gg k_{p}}(t) / \partial \ln t=f_{>}
$$

Knowledge of the time evolution for the modes on both sides of the peak of the spectrum is therefore sufficient to determine both $\mathcal{A}(t)$ and $k_{p}(t)$. (This holds as long as the parametrization (4.1) remains valid.) Indeed, one finds the simple relation

$$
\frac{\partial \ln k_{p}}{\partial \ln t}=\frac{f_{>}-f_{<}}{\nu_{I R}-\nu_{U V}}
$$

Inversely, if $k_{p}(t)$ is known, this yields a relation for the difference in the slopes, $\nu_{I R}-\nu_{U V}$. For the radiation spectrum and the infrared spectrum the growth exponent $\partial \ln \alpha_{k} / \partial \ln t$ is typically the same on both sides of the peak. Therefore $k_{p}$ remains constant as expected. We have already argued in the last section that the radiation-like spectrum indeed leads to the equation of state of relativistic matter with $q_{\varphi}=0$. The radiation-like spectrum seems not to be well motivated or interesting, however.

For the horizon-dominated spectrum the difference in the slope of the the spectrum on both sides of the peak is due to the different growth rates for short wavelength and infrared 
modes. Typically, the short-distance modes are relativistic, whereas the modes with $k$ somewhat smaller than $k_{p}$ are nonrelativistic. The growth rate of the infrared modes is also influenced by the gravitational interaction and depends, in general, on the chosen gauge of the metric. If $k_{p}$ scales with the horizon, $k_{p} \sim a H$, one has a general connection between the growth rates $f_{>}, f_{<}$and the difference in slope between the ultraviolet and infrared spectrum $\left(H=\eta t^{-1}\right)$

$$
\begin{gathered}
\frac{\partial \ln k_{p}}{\partial \ln t}=-(1-\eta), \\
\nu_{I R}-\nu_{U V}=\frac{f_{<}-f_{>}}{1-\eta}
\end{gathered}
$$

A spectrum with finite energy density $\left(\nu_{I R}-\nu_{U V}>2\right)$ requires $f_{<}-f_{>}>2(1-\eta)$.

For the purpose of later comparison, we first neglect gravity and incorporate it in the next sections. Within the approximations of the last section the conserved particle number (3.10) and the relation (3.14) implies then

$$
\frac{d}{d t}\left[a^{3}\left(\frac{k^{2}}{a^{2}}+V^{\prime \prime}\right)^{1 / 2} \alpha_{k}\right]=0
$$

such that

$$
\partial_{t} \ln \alpha_{k}=-3 H-\frac{1}{2} \partial_{t} \ln \left(\frac{k^{2}}{a^{2}}+V^{\prime \prime}\right)
$$

One infers for $k_{p}^{2} / a^{2} \sim V^{\prime \prime} \sim t^{-2}$ and $H=\eta t^{-1}$ the different growth rates

$$
\begin{aligned}
& f_{>}=\partial \ln \alpha_{k \gg k_{p}} / \partial \ln t=-2 \eta, \\
& f_{<}=\partial \ln \alpha_{k \ll k_{p}} / \partial \ln t=-(3 \eta-1)
\end{aligned}
$$

and therefore

$$
\frac{\partial \ln k_{p}}{\partial \ln t}=-\frac{1}{\nu_{I R}-\nu_{U V}}(1-\eta)
$$

On the other hand, the separation between relativistic and nonrelativistic behavior at $k_{p}^{2} \sim V^{\prime \prime} a^{2}$ corresponds to (4.10) and we conclude that the faster decay of the relativistic modes as compared to the nonrelativistic ones leads to a spectrum with

$$
\nu_{I R}-\nu_{U V}=1
$$

This difference is not enough to fulfil simultaneously both bounds in eq. (4.3).

Actually, the relation (4.12) is only obeyed for the modes well within the horizon and only the estimate of $f_{>}=-2 \eta$ is therefore reliable. We may combine the relations (4.10), (4.8), (4.7) and the growth rate for the relativistic modes (4.14) to infer

$$
f_{<}=\partial \ln \alpha_{k \ll k_{p}} / \partial \ln t=(1-\eta)\left(\nu_{I R}-\nu_{U V}\right)-2 \eta
$$


Consistency with the bounds (4.3) requires then $\nu_{I R}-\nu_{U V}>2$ or

$$
f_{<}>2-4 \eta
$$

We observe that for $\eta=\frac{1}{2}$ (as in the radiation-dominated epoch) a consistent picture for the spectrum (4.1) emerges only if the long wavelength modes grow, i.e. $f_{<}>0$. We will see in the next section how gravity effects induce a nonvanishing particle production rate $\Delta_{k}$ and modify the growth rate $f_{<}$as compared to eq. (4.15). Depending on the shape of the cosmon potential, we will see in sect. 8 that indeed $f_{<}$is zero during the radiation-dominated epoch.

Finally, we discuss for the spectrum (4.1) the time evolution of the quantities $A$ and $C$ for arbitrary $f_{<}$. One has for a horizon-dominated spectrum (cf. eq. (4.4), (4.10))

$$
\frac{\partial \ln A}{\partial \ln t}=f_{<}-(1-\eta) \nu_{I R}=-(1-\eta) \nu_{U V}-2 \eta
$$

and $A$ can only grow for positive $f_{<}>(1-\eta) \nu_{I R}$. The relevant quantity for the equation of state

$$
\frac{C}{a^{2} H^{2} A}=c_{p} \kappa^{2}
$$

is time-independent (for $k_{p}^{2} / a^{2}=\kappa^{2} H^{2}$ ). The relation (4.21) also shows that for a horizondominated spectrum the equation of state for cosmon dark matter will depend on the details of the spectrum around the maximum since the constants $c_{p}$ and $\kappa$ enter crucially.

Quite generally the details around the maximum of $k^{3} \alpha_{k}$ depend crucially on the precise understanding of the evolution of the modes with momenta near the maximum. On the other hand, the relation (4.9) between the slopes in the infrared and ultraviolet is insensitive to these details. This may be demonstrated by assuming a different growth rate and different slopes of the spectrum in three different regions, $\left(k_{1} \ll k_{2}\right)$

$$
k^{3} \alpha_{k} \sim \begin{cases}k^{\nu_{I R}} & \text { for } k \ll k_{1}(t) \\ k^{\nu_{\max }} & \text { for } k_{1}(t) \ll k \ll k_{2}(t) \\ k^{\nu_{U V}} & \text { for } k \gg k_{2}(t)\end{cases}
$$

as exemplified by

$$
\alpha_{k}=\bar{\alpha}(t) M^{2} k^{\nu_{I R}-3}\left(1+\frac{k}{k_{1}(t)}\right)^{\nu_{\max }-\nu_{I R}}\left(1+\frac{k}{k_{2}(t)}\right)^{\nu_{U V}-\nu_{\max }}
$$

Defining the growth rates $f_{<}$and $f_{>}$for the infrared and ultraviolet modes as before (e.g. eqs. (4.14), (4.15) with $k_{p}$ replaced by $k_{1}, k_{2}$ ) and

$$
f_{\max }=\partial \ln \alpha_{k_{1} \ll k \ll k_{2}} / \partial \ln t
$$


leads to the relations

$$
\begin{aligned}
& f_{\text {max }}-f_{<}=\left(\nu_{I R}-\nu_{\max }\right) \frac{\partial \ln k_{1}}{\partial \ln t} \\
& f_{>}-f_{\max }=\left(\nu_{\max }-\nu_{U V}\right) \frac{\partial \ln k_{2}}{\partial \ln t}
\end{aligned}
$$

If both $k_{1}$ and $k_{2}$ scale proportional to $k_{p}$, we indeed recover the relation (4.9) independent of the growth rate of the modes near the maximum, $f_{\max }$. This tells us that in the case where $f_{<}$does not obey the bound (4.19) another scale different from the horizon must be present in the spectrum. One possibility could be an effective ultraviolet cutoff $k_{U V}$ beyond which the spectrum is further suppressed. Due to the extremely weak interaction of the cosmon with ordinary matter such a cutoff does not arise from microscopical processes in the late universe. A natural scale for such a cutoff could be the scale which corresponds to the horizon at the end of inflation. In this case $k_{U V}$ does not depend on time. (Today $a / k_{U V}$ is then typically in the centimeter range.)

\section{$5 \quad$ Linear gravity effects}

We have seen in the preceeding section that a crucial ingredient for the understanding of the spectrum of the cosmon fluctuations and therefore also for the equation of state of cosmon dark matter is the understanding of the time evolution of the modes with wavelength around and outside the horizon. For these modes the gravitational effects become important. Indeed, the effects of the gravitational attraction become comparable in strength to the effects of the mass term $V^{\prime \prime}$ which scales typically with the Hubble parameter, $V^{\prime \prime} \sim H^{2}$. We therefore include next the effects of gravity in the linear approximation. We adopt here the "background harmonic gauge"

$$
D_{\mu} h_{\nu}^{\mu}=\frac{1}{2} \partial_{\nu} h
$$

where $D_{\mu} h^{\mu}{ }_{\nu}=\partial_{\mu} h^{\mu}{ }_{\nu}+\left(\bar{g}^{-1 / 2} \partial_{\mu} \bar{g}^{1 / 2}\right) h^{\mu}{ }_{\nu}-\bar{\Gamma}_{\mu \nu}{ }^{\lambda} h^{\mu}{ }_{\lambda}$ is the covariant derivative in the background metric $\bar{g}_{\mu \nu}$ which is also used to raise and lower the indices of $h_{\mu \nu}$. It has the property that in linear order $\left(\delta g_{\mu \nu}=h_{\mu \nu}\right)$

$$
\bar{g}^{\mu \nu} \delta \Gamma_{\mu \nu}^{\lambda}=0
$$

and therefore

$$
\delta\left(\chi ;{ }_{\mu}^{\mu}\right)=(\delta \chi) ;{ }_{\mu}^{\mu}-h^{\mu \nu} \varphi ;_{\mu \nu}
$$

We believe that the background harmonic gauge gives us a comparatively simple picture

of the physical situation. This is crucial for an understanding of the large-scale cosmon 
fluctuations and justifies the somewhat higher algebraic complexity as compared to the more usual synchronous gauge. (For the synchronous gauge the growth of the dominant mode for large $t$ does not correspond to a growing gauge-invariant fluctuation and obscures the picture.)

The gravity effects result in a modification of the microscopic evolution equation for the cosmon fluctuations $\$$ for $k \neq 0$

$$
\delta \ddot{\chi}_{k}+3 H \delta \dot{\chi}_{k}+\left(\frac{k^{2}}{a^{2}}+V^{\prime \prime}\right) \delta \chi_{k}=H \dot{\varphi}\left(\left(h_{00}\right)_{k}+h_{k}\right)-\ddot{\varphi}\left(h_{00}\right)_{k}
$$

The field equations for the metric fluctuations are derived in appendix A. In harmonic gauge the trace of the metric fluctuations, $h=h_{\mu}^{\mu}=\bar{g}^{\mu \nu} h_{\nu \mu}$, obeys (for $k \neq 0, h_{k=0} \equiv 0$ )

$$
h ;_{\mu}^{\mu}=-\frac{1}{M^{2}}\left(4 V^{\prime} \delta \chi-2 \dot{\varphi} \delta \dot{\chi}+V h+\frac{\rho+p}{2}\left(h+4 h_{00}\right)\right)
$$

We observe that contributions involving the potential of the background field $\left(\sim V, V^{\prime}\right)$ conserve the Lorentz symmetry and therefore multiply Lorentz-scalars $\delta \chi, h$. On the other hand, terms involving $\dot{\varphi}, \ddot{\varphi}$ or $\rho+p$ respect only the rotation symmetry. This explains the separate appearance of $h_{00}$.

A second effect of gravity are corrections to the energy momentum tensor and to $q_{\varphi}$ according to eq. (2.8). Using the definitons of (3.8) and similarly $q_{\varphi}=\int_{k} q_{k}$, the additional contributions $\left(\rho_{k}=\left(k^{2} / a^{2}+V^{\prime \prime}\right)^{1 / 2} n_{k}+\Delta \rho_{k}\right.$ etc.) up to second order in $h_{\mu \nu}$ are

$$
\begin{gathered}
\Delta \rho_{k}=-V^{\prime}<\delta \chi h_{00}>_{k} \\
\Delta p_{k}=-\frac{V^{\prime}}{3}<\delta \chi h_{i}^{i}>_{k}+\frac{\dot{\varphi}}{3}<\delta \dot{\chi} h_{i}^{i}>_{k} \\
+\dot{\varphi}<\delta \dot{\chi} h_{00}>_{k}+\frac{\dot{\varphi}^{2}}{2}<h_{00}^{2}>_{k}+\frac{\dot{\varphi}^{2}}{6}<h_{00} h_{i}^{i}>_{k} \\
\Delta q_{k}=<\chi_{\mu}^{\mu}>_{k}=<\frac{1}{2} \partial^{\nu} h \partial_{\nu} \delta \chi+3 H h_{0}^{\nu} \partial_{\nu} \delta \chi-\partial_{\mu} h^{\mu \nu} \partial_{\nu} \delta \chi \\
-h^{\mu \nu} \partial_{\mu} \partial_{\nu} \delta \chi>+(\ddot{\varphi}+3 H \dot{\varphi})<h_{0}{ }^{\rho} h_{\rho 0}> \\
-\dot{\varphi}<\partial_{\mu} h^{\mu \rho} h_{\rho 0}+h^{\mu \rho} \partial_{\mu} h_{\rho 0}-\frac{1}{2} \partial_{\mu} h h_{0}^{\mu}-\frac{1}{2} h_{\nu}^{\rho} h_{\rho}^{\nu}>
\end{gathered}
$$

We observe that the relations (5.6)-(5.8) do not yet use a particular gauge. Finally, the metric fluctuations lead to a pure gravitational contribution to the energy momentum tensor $\rho_{g}, p_{g}$ (cf eq. (2.9)). This is discussed in appendix B.

\footnotetext{
${ }^{9}$ Note that one has to linearize precisely the equation $\left(\chi \dot{*}_{\mu}^{\mu}-V^{\prime}\right)_{k}=\delta\left(\chi_{;_{\mu}}^{\mu}\right)_{k}-\left(\delta V^{\prime}\right)_{k}-\left(q_{\varphi}\right)_{k}=0$, using $\left(q_{\varphi}\right)_{k \neq 0}=0$.)
} 
We need the relative size of $h_{i}^{i}=h_{i i} / a^{2}$ and $h_{00}$ with $h=-h_{00}+h_{i}^{i}$. This issue is discussed in appendix A. For many purposes the ratio

$$
y_{k}=\frac{\left(h_{00}\right)_{k}}{h_{k}}
$$

can be taken as a time-independent (complex) constant. (This holds for $V^{\prime} / \dot{\varphi}=$ const and $t \delta \dot{\chi}_{k} \sim \delta \chi_{k}$, with complex proportionality factor for oscillating $\delta \chi_{k}$.) One obtains the microscopic equation for the trace of the metric fluctuations

$$
\ddot{h}_{k}+3 H \dot{h}_{k}+\left(\frac{k^{2}}{a^{2}}-\frac{V}{M^{2}}-\frac{\rho+p}{2 M^{2}}\left(1+4 y_{k}\right)\right) h_{k}=\frac{1}{M^{2}}\left(4 V^{\prime} \delta \chi_{k}-2 \dot{\varphi} \delta \dot{\chi}_{k}\right)
$$

This is our central equation for the time evolution of $h$. We note that it continues to hold in presence of additional radiation fluctuations which obey $\delta \rho_{r}-3 \delta p_{r}=0$ and do not contribute to $\delta t_{\rho}^{\rho}=\bar{g}^{\rho \lambda} \delta t_{\rho \lambda}$. Besides the contribution to $\rho+p$, additional radiation can therefore only affect the value of $y_{k}$.

For large enough $k$ we can neglect the retardation effects (i.e. neglect the time derivatives of $h_{k}$ ) and approximate

$$
h_{k}=-\frac{a^{2}}{M^{2} k^{2}}\left(\delta t_{\mu}^{\mu}\right)_{k}=\frac{4 a^{2} V^{\prime}}{M^{2} k^{2}} \delta \chi_{k}-\frac{2 a^{2} \dot{\varphi}}{M^{2} k^{2}} \delta \dot{\chi}_{k}
$$

The r.h.s. of eq. (5.4) therefore becomes

$$
H \dot{\varphi}\left(h_{k}+\left(h_{00}\right)_{k}\right)-\ddot{\varphi}\left(h_{00}\right)_{k}=\left[H \dot{\varphi}+(H \dot{\varphi}-\ddot{\varphi}) y_{k}\right]\left(\frac{4 a^{2} V^{\prime}}{M^{2} k^{2}} \delta \chi_{k}-\frac{2 a^{2} \dot{\varphi}}{M^{2} k^{2}} \delta \dot{\chi}_{k}\right)
$$

and the modified microscopic equation reads

$$
\delta \ddot{\chi}_{k}+\left\{3 H+\frac{2 u_{k} H \dot{\varphi}^{2} a^{2}}{M^{2} k^{2}}\right\} \delta \dot{\chi}_{k}+\left\{\frac{k^{2}}{a^{2}}+V^{\prime \prime}-\frac{4 u_{k} H \dot{\varphi} V^{\prime} a^{2}}{M^{2} k^{2}}\right\} \delta \chi_{k}=0
$$

with

$$
u_{k}=1+y_{k}-\frac{y_{k} \ddot{\varphi}}{H \dot{\varphi}}
$$

We observe a time-dependent critical value of $k$,

$$
k_{c}^{2}(t)=\frac{a^{2} \dot{\varphi}^{2}}{M^{2}}=O\left(a^{2} H^{2}\right)
$$

such that for $k^{2} \gg k_{c}^{2}$ the gravitational corrections become negligible맘 For the modes in this momentum range the results of the last section apply and the validity of the

\footnotetext{
${ }^{10}$ The details of this estimate depend on the time evolution of $V^{\prime}, V^{\prime \prime}$.
} 
approximation (5.11) can be checked by an iterative solution. For a large class of cosmon potentials one has $\dot{\varphi}^{2} \sim t^{-2}$ and $k_{c}^{2}$ decreases with time such that the range of "relativistic modes" $k>k_{c}(t)$ increases. On the other hand, for the long wave-length modes with $k^{2} \lesssim k_{c}^{2}$ the attraction by gravity becomes important. We will investigate these modes in sect. 7 .

\section{Quintessence with exponential potential}

The details of the gravitational effects depend on the particular quintessence model, i.e. the time history of $V^{\prime}, V^{\prime \prime}$ and $\dot{\varphi}$. For an illustration, we take here an exponential potential

$$
V(\varphi)=M^{4} \exp \left(-\alpha \frac{\varphi}{M}\right)
$$

In addition to cosmon dark matter we also allow for an independent radiation component from other particles. The combined energy density and pressure from radiation and cosmon dark matter will be denoted by $\bar{\rho}=\rho_{r}+\rho$ and similarly $\bar{p}=\frac{1}{3} \rho_{r}+p$. We also assume (cf. eq. (3.19)

$$
\bar{q}_{\varphi}=\frac{\bar{\beta}}{M} \bar{\rho}
$$

with constant $\bar{\beta}$. An asymptotic solution for $3 \bar{p}=(\bar{n}-3) \bar{\rho}$ with constant $\bar{n}$ was found in [1], 田]

$$
\begin{aligned}
H & =\eta t^{-1}, \quad \dot{\varphi}=\frac{2 M}{\alpha} t^{-1}, \quad \ddot{\varphi}=-\dot{\varphi} t^{-1}, \quad V^{\prime \prime}=\zeta_{0} t^{-2} \\
V^{\prime} & =-\frac{\alpha}{M} V=-\frac{M}{\alpha} V^{\prime \prime}=-\frac{M \zeta_{0}}{\alpha} t^{-2}
\end{aligned}
$$

Such a solution requires

$$
\eta=\frac{2}{\bar{n}}\left(1-\frac{\bar{\beta}}{\alpha}\right)
$$

and

$$
\zeta_{0}=\frac{2}{\bar{n}^{2}}\{\bar{n}(6-\bar{n})-12 \bar{\beta}(\alpha-\bar{\beta})\}
$$

Inserting these values yields algebraic relations for the energy density in particles (other than the cosmon) $\Omega_{p}=\bar{\rho} / \rho_{c r}$, the scalar potential $\Omega_{V}=V / \rho_{c r}$ and homogenous quintessence $\Omega_{h}=\left(V+\frac{1}{2} \dot{\varphi}^{2}\right) / \rho_{c r}\left(\right.$ with $\left.\rho_{c r}=6 M^{2} H^{2}\right)$

$$
\begin{aligned}
\Omega_{p} & =1-\frac{\bar{n}-2 \bar{\beta}(\alpha-\bar{\beta})}{2(\alpha-\bar{\beta})^{2}} \\
\Omega_{V} & =\frac{\bar{n}(6-\bar{n})-12 \bar{\beta}(\alpha-\bar{\beta})}{12(\alpha-\bar{\beta})^{2}}=\frac{\zeta_{0}}{6 \alpha^{2} \eta^{2}} \\
\Omega_{h} & =1-\Omega_{p}
\end{aligned}
$$


We note that homogenous quintessence $\left(\rho_{h}, p_{h}\right)$ receives contributions both from the potential and the kinetic energy of the scalar background field $\varphi$. The positivity of $\Omega_{p}$ and $\Omega_{V}$ imposes the bounds

$$
\begin{aligned}
\alpha(\alpha-\bar{\beta}) & >\frac{\bar{n}}{2} \\
\bar{\beta}(\alpha-\bar{\beta}) & <\frac{\bar{n}(6-\bar{n})}{12}
\end{aligned}
$$

We will concentrate on three special cases:

a) Radiation domination

Let us first consider a situation where cosmon dark matter is subdominant as compared to the dominant radiation from other particles. In this case one has $\bar{n}=4$ and $\bar{\beta}=0$ such that the solution exists for $\alpha^{2}>2$. One finds

$$
\eta=\frac{1}{2}, \quad \zeta_{0}=1, \quad \Omega_{h}=\frac{2}{\alpha^{2}}
$$

b) Cosmon dark matter dominated by large-scale fluctuations

Next we investigate a scenario where the inhomogenous energy density is dominated by cosmon fluctuations such that $\bar{n}=n, \bar{\beta}=\beta$. We assume that the properties of cosmon dark matter can be approximately described by the large-scale cosmon fluctuations. In this case the term $\sim C$ in eq. (3.1) can be neglected and one finds

$$
\left(q_{\varphi}-\Delta q_{\varphi}\right)=\frac{\alpha}{2 M}(\rho-p-\Delta \rho+\Delta p)
$$

Without the gravity corrections (5.6), i.e. for $\Delta q_{\varphi}=\Delta \rho=\Delta p=0$, this would yield

$$
\beta=\frac{6-n}{6} \alpha
$$

and therefore

$$
\eta=\frac{1}{3} \quad, \quad \zeta_{0}=\frac{2(6-n)}{n}\left(1-\frac{\alpha^{2}}{3}\right)
$$

In this approximation the bounds (6.7) would require simultaneously $\alpha^{2}>3$ and $\alpha^{2}<3$ and we could consider the solution at best as a limiting case where the cosmon kinetic energy dominates. We will argue, however, that the gravity corrections (5.6) need to be included and therefore the relation (6.10) does not hold. For the time being we will consider $\beta$ and $n$ as free parameters characterizing the incoherent fluctuations. We will discuss in sect. 9 how to determine them self-consistently. 
c) Cosmon domination in absence of particle production

Finally we investigate a situation where the inhomogenous energy density is dominated by cosmon dark matter in the absence of particle production. This last scenario is somewhat hypothetical and may apply in a situation where the cosmon fluctuations are already nonlinear and wave lengths inside the horizon dominate, without showing the characteristics of a relativistic gas. An example may be cosmon lumps [19]. For this case one has again $\bar{n}=n$ and $\bar{\beta}=\beta$. Omitting the corrections (5.6) one has in absence of particle production $\left(\Delta_{k}=0\right.$, cf. eq. $\left.(3.20)\right)$

$$
\beta=\frac{\alpha}{2}(4-n), \quad \eta=\frac{2}{n}\left(1-\frac{\beta}{\alpha}\right)=1-\frac{2}{n}
$$

and

$$
\begin{aligned}
V^{\prime \prime} & =\frac{\alpha^{2}}{M^{2}} V=\frac{2 n(6-n)-6 \alpha^{2}(4-n)(n-2)}{(n-2)^{2}} H^{2} \\
& =\frac{2}{n^{2}}\left\{n(6-n)-3 \alpha^{2}(4-n)(n-2)\right\} t^{-2}=\zeta_{0} t^{-2},
\end{aligned}
$$

We emphasize the unusual expansion rate for $n \neq 4$ due to the coupling $\beta$. In particular, for the equation of state of nonrelativistic matter, $n=3$, we find $\eta=1 / 3$ and therefore $a \sim t^{1 / 3}$ instead of the usual $a \sim t^{2 / 3}$. The relative contribution to the energy density of cosmon dark matter is given for this solution by

$$
\Omega_{p}=\frac{2}{n-2}\left(1-\frac{n}{\alpha^{2}(n-2)}\right)
$$

whereas homogenous quintessence accounts for the rest, $\Omega_{h}=1-\Omega_{p}$. According to eq. (6.7), this solution exists only for

$$
\begin{gathered}
\alpha^{2}>\frac{n}{n-2} \\
\alpha^{2}<\frac{n(6-n)}{3(4-n)(n-2)}
\end{gathered}
$$

which requires $n>3$. At the upper bound (6.16) for $\alpha^{2}$ the potential energy $V$ goes to zero whereas at the lower bound (6.15) the incoherent energy density $\rho$ vanishes and cosmology becomes dominated by the homogenous scalar field or quintessence. The bounds coincide for $n=3$ where the "stability range" for $\alpha$ shrinks to the point $\alpha^{2}=3$. We note that for $\alpha$ in the range $2<\alpha^{2}<3$ the above solution is stable for $n=4$ but may shift to a quintessence dominated universe as $n$ decreases below a critical value, for example at matter-radiation equality. Such a behavior may lead to an interesting interpretation of present cosmological observations.

Even though not all of the cases sketched here are realistic, it becomes apparent that the presence of cosmon dark matter could have an important backreaction effect on the evolution of homogenous quintessence with nontrivial modifications of cosmology. 


\section{$7 \quad$ Growth of cosmon fluctuations?}

In this section we turn to the evolution of the large-scale cosmon fluctuations. We adopt the particular exponential quintessence potential discussed in the last section. We have seen previously that the cosmon fluctuations well within the horizon behave as relastivistic matter. These short wavelength fluctuations simply add to other forms of relativistic matter. Even if they would dominate the energy density, they would behave similar to standard radiation since $n=4$ and $\beta=0$. (For the short-distance modes the neglection of $\Delta \rho_{k}, \Delta p_{k}, \Delta q_{k}$ (cf. eq. (5.6)) is justified.) In particular, the relativistic cosmon fluctuations do not grow as compared to $\rho$.

A more interesting behavior may be expected for modes with wavelength larger than, or comparable to, the horizon. There the gravity effects become important. We cannot neglect the retardation effects and have to solve the coupled system of equations for the cosmon and gravity. With $\tau=\ln t$ eq. (5.10) reads

$$
\begin{aligned}
\partial_{\tau}^{2} h_{k} & +(3 \eta-1) \partial_{\tau} h_{k}+\left(\frac{\bar{n}}{6 \alpha^{2}}\left(2+\zeta_{0}-6 \eta^{2} \alpha^{2}\right)\left(1+4 y_{k}\right)-\frac{\zeta_{0}}{\alpha^{2}}\right) h_{k}+\frac{k^{2} t^{2}}{a^{2}} h_{k} \\
& =-\frac{4}{M \alpha}\left(\zeta_{0} \delta \chi_{k}+\partial_{\tau} \delta \chi_{k}\right)
\end{aligned}
$$

and similarly for the scalar fluctuations eq. (5.4) becomes

$$
\partial_{\tau}^{2} \delta \chi_{k}+(3 \eta-1) \partial_{\tau} \delta \chi_{k}+\zeta_{0} \delta \chi_{k}+\frac{k^{2} t^{2}}{a^{2}} \delta \chi_{k}=\frac{2 M}{\alpha} h_{k}\left(y_{k}+\eta+\eta y_{k}\right)
$$

For the modes far outside the horizon we can neglect the terms $\sim k^{2} t^{2} / a^{2}$. The resulting system of linear differential equations has time-independent coefficients for the case of the quintessence background discussed in the preceeding section. It can be reduced to a system of first-order differential equations and solved by standard methods. The eigenvectors of the fluctuation matrix obey

$$
\delta \chi_{k}(\tau)=\delta \chi_{k}(0) e^{\lambda \tau}, \quad h_{k}(\tau)=\frac{4 s_{0}}{\alpha M} \delta \chi_{k}(\tau)
$$

where the constants $\lambda$ and $s_{0}$ obey

$$
\begin{aligned}
& s_{0} \lambda^{2}+(3 \eta-1) s_{0} \lambda+x s_{0}+\zeta_{0}+\lambda=0 \\
& \lambda^{2}+(3 \eta-1) \lambda+\zeta_{0}-\frac{8}{\alpha^{2}} s_{0} w=0
\end{aligned}
$$

and

$$
x=\frac{\bar{n}}{6 \alpha^{2}}\left(2+\zeta_{0}-6 \eta^{2} \alpha^{2}\right)\left(1+4 y_{k}\right)-\frac{\zeta_{0}}{\alpha^{2}} \quad, \quad w=y_{k}+\eta+\eta y_{k}
$$


With

$$
s_{0}=\frac{\alpha^{2}}{8 w}\left(\lambda^{2}+(3 \eta-1) \lambda+\zeta_{0}\right)
$$

the growth exponent $\lambda$ obeys the "quartic" equation"

$$
\lambda^{4}+2(3 \eta-1) \lambda^{3}+\left\{\zeta_{0}+x+(3 \eta-1)^{2}\right\} \lambda^{2}+\left\{(3 \eta-1)\left(\zeta_{0}+x\right)+\frac{8 w}{\alpha^{2}}\right\} \lambda+v=0
$$

with

$$
v=\zeta_{0}\left(x+\frac{8 w}{\alpha^{2}}\right)
$$

and we have to select the solution for $\lambda$ with the largest real part. We note that eq. (7.7) has at least one positive real solution if $v$ is negative. A positive eigenvalue of $\lambda$ would imply that the large scale fluctuations grow $\sim t^{\lambda}$. Such fluctuations would be a good candidate for becoming nonlinear.

In appendix A we have evaluated $y_{k}$ for the modes far outside the horizon. We discuss possible solutions where $h_{0}^{i}$ remains small for $k \rightarrow 0$. Then $y_{k}$ depends on $\lambda$ whereas it becomes independent of $k$ :

$$
y=-(\lambda+2 \eta) /(2 \lambda+8 \eta)
$$

For large $|\lambda|$ it approaches a constant, $y \rightarrow-1 / 2$, whereas for $\lambda \rightarrow 0$ one finds $y \rightarrow-1 / 4$. We have checked the validity of the assumption leading to eq. (77.9) for $\lambda=0$ (see next sect.). (In any case, there is no reason that $y$ becomes unbounded for $\lambda \rightarrow \infty$.) We conclude that for large $\lambda$ the expression (7.7) grows $\sim \lambda^{4}$ whereas for $\lambda \rightarrow 0$ it approaches a constant

$$
v_{0}=v(y(\lambda=0))=-\frac{\zeta_{0}}{\alpha^{2}}\left(\zeta_{0}+2(1-3 \eta)\right)=\frac{12 \zeta_{0} \bar{\beta}}{\alpha^{3} \bar{n}}\left(\alpha^{2} \eta-1\right)
$$

which is negative for negative $\bar{\beta}$. For $\bar{\beta}<0$ the existence of a positive eigenvalue is therefore indeed guaranteed. For $\bar{\beta}=0$ there is at least a constant mode $(\lambda=0)$ and there may be an additional growing mode (cf. sect. 8).

Quite generally, the evolution of the modes far outside the horizon is characterized by a nonvanishing "particle production rate" $\Delta_{k}$ in the sense of sect. 3. From

$$
\delta \dot{\chi}_{k}=\frac{\lambda}{t} \delta \chi_{k}
$$

one infers

$$
\beta_{k}=\frac{\lambda^{2}}{t^{2}} \alpha_{k}
$$

Inserting this behavior in eqs. (3.6), (3.7), and using $V^{\prime \prime}=\zeta_{0} t^{-2}$ (6.3), we obtain

$$
\Delta_{k}=\frac{\zeta_{0}-\lambda^{2}}{\zeta_{0}+\lambda^{2}} n_{k}
$$

\footnotetext{
${ }^{11}$ Note that $y_{k}$ and therefore $x$ and $w$ depend on $\lambda$ such that eq. (7.7) is not simply a polynomial in $\lambda$.
} 
and therefore (cf. eq. (3.9) ) for the equation of state

$$
p_{k}=-\frac{\Delta_{k}}{n_{k}}\left(\rho_{k}-\Delta \rho_{k}\right)+\Delta p_{k}=\frac{\lambda^{2}-\zeta_{0}}{\lambda^{2}+\zeta_{0}}\left(\rho_{k}-\Delta \rho_{k}\right)+\Delta p_{k}
$$

\section{Cosmon fluctuations in a radiation-dominated universe}

Let us consider the evolution of the cosmon modes outside the horizon in a radiationdominated universe. We assume here that at some early time the fluctuation amplitudes are small such that the linear approximation applies. We want to know if the energy density of the cosmon fluctuations can grow relative to the radiation such that the cosmons finally dominate at large time. The fluctuation analysis of the preceeding sections can easily be extended to this case. In particular, the evolution equation (5.5) for $h$ remains unchanged. Fluctuations in the radiation do not contribute to $\delta t_{\mu}^{\mu}$ due to $\delta p_{r}=\delta \rho_{r} / 3$. We investigate here a possible solution where for $k \rightarrow 0$ the fluctuations $h_{0}^{i}$ can be neglected compared to $h$ or $h_{00}$. In this case also the ratio $y=h_{00} / h$ becomes independent of additional radiation fluctuations in the limit $k^{2} /\left(a^{2} H^{2}\right) \rightarrow 0$. (The estimate (A.28) only involves the smallness of $h_{0 i}$ as compared to $h$. For the modes inside the horizon the ratio $y_{k}$ will depend on an additional radiation component.) For a radiation-dominated universe the background solution obeys $\bar{n}=4, \bar{\beta}=0, \eta=1 / 2, \zeta_{0}=1$. This yields

$$
\begin{aligned}
& y=-\frac{1+\lambda}{4+2 \lambda} \quad, \quad x=-\frac{2+3 \lambda-\alpha^{2} \lambda}{\alpha^{2}(2+\lambda)}, \\
& w=\frac{1-\lambda}{8+4 \lambda} \quad, \quad v_{0}=0
\end{aligned}
$$

and implies the existence of a solution with stationary large-scale cosmon fluctuations, $\lambda=0$. In addition, eq. (7.7) with eq. (7.9) has a solution with a positive real $\lambda$ for

$\alpha^{2}<2$. We concentrate here on the case where the fluctuations far outside the horizon $(k \rightarrow 0)$ are constant, i.e. $\lambda=0$.

For the constant solution $(\lambda=0)$ we find $\left(x=-1 / \alpha^{2}, w=1 / 8, s_{0}=\alpha^{2}\right)$

$$
h_{k}=\frac{4 \alpha}{M} \delta \chi_{k}, \quad\left(h_{00}\right)_{k}=-\frac{\alpha}{M} \delta \chi_{k}
$$

In eq. (7.2) the gravitational attraction precisely compensates the driving force due to the potential. It is easy to check with eq. (A.23) that for this particular solution the linear fluctuations in the energy momentum tensor vanish

$$
\delta \rho_{k}=\delta p_{k}=0
$$


In consequence, the evolution equations for the linear metric fluctuations (A.17), (A.18) have no source term $\delta s_{00}=0, \delta s_{\mu}^{\mu}=0$. This allows indeed a constant solution for the metric provided $h_{00}=-h / 4$ (cf. eq. (A.18)). Furthermore, eq. (8.3) gives a nice example that nonvanishing cosmon fluctuations $\delta \chi_{k}$ lead not always to energy density fluctuations in linear order. This is particularly important for small positive $\lambda$ since by continuity we may expect an approximate decoupling between the growth of $\delta \chi_{k}$ and $\delta \rho_{k}$. This may be relevant for reconciling comparatively large cosmon fluctuations with very small fluctuations in the radiation.

A simple picture for the cosmon fluctuations in the radiation-dominated era emerges: For the modes outside the horizon the fluctuations $\delta \chi_{k}$ and therefore also $\alpha_{k}$ remain constant. Their contribution to the energy momentum tensor scales $\sim V$ and therefore decreases $\sim t^{-2}$, just as the energy density in radiation and quintessence. On the other hand, the modes well inside the horizon decrease in amplitude according to

$$
\partial \ln \alpha_{k \gg k_{p}} / \partial \ln t=f_{>}=-1
$$

implying $\alpha_{k \gg k_{p}} \sim t^{-1}$. Their contribution to the energy momentum tensor is dominated by the term $\sim k^{2} \alpha_{k} / a^{2}$ (the contribution $\sim C / a^{2}$ in eq. (3.1)) and therefore also decreases $\sim t^{-2}$. In short, the cosmon dark matter scales proportional to the relativistic matter, just as the homogenous quintessence contribution.

Using the relations (8.2) we can also evaluate the equation of state for the long wavelength fluctuations ${ }^{\mathbb{2}}$. Since the linear contribution vanishes according to eq. (8.3), the lowest order contribution is quadratic in the fluctuations. We find that the mixed cosmon gravity contribution $\Delta \rho, \Delta p$ dominates $\left(\rho_{k}=\rho_{k}^{(\delta \chi)}+\Delta \rho_{k}\right.$ etc. $)$

$$
\begin{aligned}
\rho_{k}^{(\delta \chi)} & =\frac{\zeta_{0}}{2 t^{2}} \alpha_{k}, \quad p_{k}^{(\delta \chi)}=-\rho_{k}^{(\delta \chi)} \\
(\Delta \rho)_{k} & =-2 \rho_{k}^{(\delta \chi)}, \quad \Delta p_{k}=2 \rho_{k}^{(\delta \chi)} \\
q_{k}^{(\delta \chi)} & =\frac{\alpha \zeta_{0}}{2 M} \frac{\alpha_{k}}{t^{2}}, \Delta q_{k}=-2 q_{k}^{(\delta \chi)}
\end{aligned}
$$

In particular, the large wavelength cosmon fluctuations give a negative contribution ${ }^{\text {T3 }}$ to the energy density!

$$
\begin{aligned}
\left(\rho_{c}\right)_{k} & =-\left(p_{c}\right)_{k}=-\frac{\alpha_{k}}{2 t^{2}}, \\
\left(q_{\varphi}\right)_{k} & =-\frac{\alpha}{M} \frac{\alpha_{k}}{2 t^{2}}
\end{aligned}
$$

For the equation of state for the long-distance modes we find $\left(p_{c}\right)_{k} /\left(\rho_{c}\right)_{k}=-1$ and the reader may wonder how this is compatible with a scaling $\left(\rho_{c}\right)_{k} \sim t^{-2}$. The solution of this

\footnotetext{
${ }^{12}$ Notice that for $\lambda=0$ one has $\beta_{k}=0$ and therefore $\Delta_{k}=n_{k}$.

${ }^{13}$ Here we use $\zeta_{0}=1$ as appropriate for the radiation-dominated universe.
} 
puzzle arises from the term $q_{\varphi}$ in the cosmon field equation. Indeed, the relation (2.10)

$$
\left(\dot{\rho}_{c}\right)_{k}+3 H\left(\left(\rho_{c}\right)_{k}+\left(p_{c}\right)_{k}\right)+\dot{\varphi}\left(q_{\varphi}\right)_{k}=0
$$

is obeyed separately for every $k$-mode in the linear approximation. For large $k$ we have a characteristic relativistic equation of state $\left(p_{c}\right)_{k}=\frac{1}{3}\left(\rho_{c}\right)_{k}$ and $\left(q_{\varphi}\right)_{k}=0$ whereas for low $k$ the relations (8.6) obey again (8.7) for $\left(\rho_{c}\right)_{k} \sim t^{-2}$ due to the nonvanishing $\left(q_{\varphi}\right)_{k}$. While for large $k$ the energy density $\left(\rho_{c}\right)_{k} \approx \rho_{k}^{(\delta \chi)}$ is positive and decreases due to the expansion as usual, the negative energy density for small $k$ increases as a result of an intricate interplay between gravity and the scalar equation of motion! For the convenience of the reader we summarize in table 1 the time behavior of some relevant characteristic quantities for the radiation-dominated era.

\begin{tabular}{|c|c|}
\hline small $k$ & large $k$ \\
\hline$\delta \chi_{k} \sim$ const & $\delta \chi_{k} \sim t^{-1 / 2}$ \\
$\alpha_{k} \sim$ const & $\alpha_{k} \sim t^{-1}$ \\
$V^{\prime \prime} \sim t^{-2}$ & $k^{2} / a^{2} \sim t^{-1}$ \\
$\rho_{k} \sim t^{-2}$ & $\rho_{k} \sim t^{-2}$ \\
$p_{k}=-\rho_{k}$ & $p_{k}=\frac{1}{3} \rho_{k}$ \\
\hline
\end{tabular}

Table 1: Time dependence of characteristic quantities in the radiation-dominated era

In addition, for the modes with small $k$ there is a relevant pure gravitational contribution to the energy momentum tensor. It is evaluated in appendix B. We find that $\rho_{g}$ and $p_{g}$ are of the same order as $\rho_{c}, p_{c}$ or smaller. We observe that nonzero $\rho_{g}, p_{g}$ also decrease $\sim t^{-2}$. Since the cosmon energy momentum tensor is separately conserved (cf. eq. (8.7)), this must also hold for the pure gravitational energy momentum tensor. In the pure gravitational sector there is no piece corresponding to $q_{\varphi}$. Therefore the decrease $\rho_{g} \sim t^{-2}$ implies that the pure gravitational part behaves similar to a relativistic gas, $p_{g}=\rho_{g} / 3$.

As a direct consequence of eq. (2.10) and $\rho_{c} \sim t^{-2}$ we can relate $q_{\varphi}=\left(\beta_{c} / M\right) \rho_{c}$ to the equation of state for cosmon dark matter

$$
\beta_{c}=\alpha\left(1-\frac{3 \eta}{2}\left(1+\frac{p_{c}}{\rho_{c}}\right)\right)=\alpha\left(1-\frac{\eta}{2} n\right)
$$

In our case this is realized for $\eta=1 / 2$, yielding

$$
\beta_{c}=\frac{\alpha}{4}(4-n)
$$


In dependence on the precise shape of the cosmon spectrum $\beta_{c}$ varies between 0 and $\alpha$ since $-\rho_{c} \leq p_{c} \leq \rho_{c} / 3$. The relation (8.9) differs from the result (3.20) (as obtained for $\left.\Delta_{k}=0\right)$ by a factor of two and coincidence occurs only for $p_{c}=\rho_{c} / 3(n=4)$. This reflects the fact that an unusual equation of state for cosmon dark matter is related in our case to the effects of gravity for the long distance modes for which $\Delta_{k} \neq 0$. We emphasize, however, that $\beta_{c}$ does not necessarily coincide with $\beta$ as defined by eq. (3.19). Whenever the total energy density $\rho$ is positive, the second equation in eq. (8.6) implies a negative value for $\beta$ !

With $f_{<}=2 \lambda$ and $\eta=1 / 2$ we can investigate a cosmon spectrum of the type discussed in sect. 4. For the solution with time-independent large-scale fluctuations $(\lambda=0)$ it is at best at the "borderline of convergence" (cf. eq. (4.19)). Indeed, from eq. (4.18) with $f_{<}=0$ we obtain

$$
\nu_{I R}-\nu_{U V}=2
$$

For $\nu_{I R}=0, \nu_{U V}=-2$ the integral (3.8) for the energy density would diverge logarithmically both for small and large $k$. On the other hand, for a small positive $\nu_{I R}>0$ the spectrum becomes infrared finite at the expense of a power divergence in the ultraviolet. As discussed at the end of sect. 4, this divergence may be cut off at a fixed scale $k_{U V}$ (which may be related to the physics of inflation). We may then visualize cosmon dark matter as an effective mixture of two components. The first "radiation component" accounts for the modes well within the horizon, say $k>k_{2}(t), k_{2}(t)=100 k_{p}(t)$. If the UV-cutoff occurs when the fluctuations remain still linear, the "radiation component" indeed behaves like relativistic matter to high accuracy, being dominated by modes with $k \approx k_{U V}$. The second "horizon component" accounts for the rest, whereby the integrals for its contribution to the energy density etc. are cut off at $k_{2}$. If the growth exponent for the modes around the horizon (corresponding to $f_{\max }$ in sect. 4) would be negative or zero, $f_{\text {max }} \leq 0$, cosmon dark matter would always be dominated by its radiation component. However, our finding that the growth rate $f_{<}$is zero for the very long distance modes could well be compatible with a positive $f_{\max }$ as well. (The computation of $f_{\max }$ is involved and not done here.) A positive value of $f_{\max }$ would have important implications. Indeed, the relative weight of the horizon component as compared to the radiation component scales ${ }^{\text {th }} \sim\left(k_{p}^{2} / k_{U V}^{2}\right)\left(\alpha_{k_{p}} / \alpha_{k_{U V}}\right) k_{p}^{\nu_{I R}}$. It grows for a large enough growth rate of the modes near the horizon, $f_{H}=\partial \ln \alpha_{k_{p}} / \partial \ln t$, namely if $f_{H}$ obeys

$$
f_{H}>f_{>}-\left(2+\nu_{I R}\right) \frac{\partial \ln k_{p}}{\partial \ln t} \quad, \quad f_{H}>2-4 \eta+\nu_{I R}(1-\eta)
$$

For the radiation-dominated era this requires $f_{H}>\nu_{I R} / 2$. The energy density in the horizon component of cosmon dark matter would then decrease only as

$$
\rho_{c, H} \sim t^{2 \lambda_{H}-\nu_{I R}-2}
$$

\footnotetext{
${ }^{14}$ The factor $k_{p}{ }^{\nu_{I R}}$ (cf. eq. (4.4)) appears only if $k^{3} \rho_{k}$ develops a maximum near $k_{p}$ due to the growth of the fluctuations at this scale.
} 
Since the homogenous radiation scales $\sim t^{-2}$, a positive value of $f_{H}-\nu_{I R} / 2$ would lead to a situation where the horizon component of cosmon dark matter finally not only overwhelms the radiation component of cosmon dark matter, but the total energy density in radiation as well! When this happens, the radiation-dominated era would end and transite to an era dominated by the horizon component of cosmon dark matter!

We emphasize that due to the negative value (8.6) of $q_{\varphi}$ the value of $\bar{\beta}$ is actually negative in a radiation-dominated universe. This effect is tiny as long as radiation dominates substantially. Once the horizon component of cosmon dark matter becomes more important, however, the size of $|\bar{\beta}|$ increases. In turn, this leads to a larger value of $\lambda$ (cf. eq. (7.10) ) and further increases the growth of the cosmon fluctuations around and beyond the horizon! We also recall at this point that quintessence with an exponential potential may be an oversimplified example. It is well conceivable [1] that the effective value of $\alpha$ depends on $\varphi$ and therefore on time. If $\alpha$ decreases with time, an increase of the horizon component of cosmon dark matter becomes even more plausible.

In conclusion, we have sketched here a first plausible scenario where cosmon dark matter grows relative to radiation. In this scenario the cosmon spectrum has the qualitative shape (4.1) with a finite energy density. Cosmon dark matter will finally overwhelm the radiation and bring the radiation-dominated epoch to an end. Also the cosmon fluctuations grow nonlinear during this evolution. Equally interesting, but more difficult to treat, is the case where the effective UV- (for $\nu_{I R}>0$ ) or $I R$ - (for $\nu_{I R}<0$ ) cutoff arises as a consequence of nonlinearities. The effective equation of state for cosmon dark matter requires then a quantitative understanding of nonlinear fluctuations.

\section{Cosmon-dominated universe}

In this section we briefly discuss a universe where homogenous quintessence and the cosmon fluctuations dominate the energy momentum tensor. Such a scenario could be realized in the "recent" past of our universe (say for $z<\left(10^{2}-10^{3}\right)$ ). The different relevant components of such a universe are gravity, homogenous quintessence, (the energy density of the scalar background field) and cosmon dark matter (the incoherent part of the expectation value of the scalar energy density). In principle, there are two possibilities: either the scaling law of cosmon dark matter is similar to the usual behavior with $\rho \sim t^{-2}$, or even the scaling law is modified. For the first case we may look for a solution of the type discussed in sect. 6 . In this case we have to determine self-consistently

$$
\begin{aligned}
& \bar{n}=n=3\left(1+\left(p_{c}+p_{g}\right) /\left(\rho_{c}+\rho_{g}\right)\right) \\
& \bar{\beta}=\beta=M q_{\varphi} /\left(\rho_{c}+\rho_{g}\right)
\end{aligned}
$$

We require $\rho=\rho_{c}+\rho_{g}>0$ and infer from eq. (8.6) that $\bar{\beta}$ is different from zero and negative. 
The computation of $\bar{n}$ and $\bar{\beta}$ is rather involved and should be done in a self-consistent way. First one has to evaluate $p, \rho$ and $q_{\varphi}$ by performing the fluctuation analysis for a family of background solutions characterized by $\bar{\beta}$ and $\bar{n}$. Then one has to solve the "self-consistency equation" (9.1) for $\bar{\beta}$ and $\bar{n}$. The solution will correspond to $\bar{\beta}<0$ and we also expect $\bar{n}<4$. In view of the linear fluctuation analysis of sec. 7 the existence of such a solution with $\lambda_{H}>0$ seems not unlikely. In this respect the precise shape of the cosmon spectrum will matter. Indeed, the relation for the growth rate of the cosmon energy density

$$
\partial \ln \rho_{c} / \partial \ln t=\partial \ln A / \partial \ln t-2=2 \lambda_{H}-(1-\eta) \nu_{I R}-2=-2
$$

implies

$$
\lambda_{H}=(1-\eta) \nu_{I R} / 2
$$

This scenario is not the only possibility and the time evolution of the various components of the energy denstiy can have a much richer structure. In the remainder of this section we summarize a few generic features of a cosmon-dominated universe. This discussion applies quite generally, far beyond the specific models discussed in this paper. In presence of quintessence and substantial cosmon fluctuations the composition of the energy momentum tensor becomes fairly complex. For a cosmon-dominated universe one may approximate $\rho_{t o t}=\rho_{h}+\rho_{\text {inhom }}=\rho_{h}+\rho_{c}+\rho_{g}$. The general characteristics of a cosmon-dominated universe can be summarized as follows:

(i) The total energy momentum tensor being conserved, i.e.

$$
\dot{\rho}_{t o t}+3 H\left(\rho_{t o t}+p_{t o t}\right)=0
$$

we can infer from eq. (2.12) the "rate of exchange" between homogenous and inhomogenous quintessence. (We use $\dot{\varphi} \geq 0$.)

$$
\begin{aligned}
& \dot{\rho}_{h}+3 H\left(\rho_{h}+p_{h}\right)=q_{\varphi} \dot{\varphi}=\frac{\beta}{M} \rho_{\text {inhom }} \sqrt{\rho_{h}+p_{h}} \\
& \dot{\rho}_{\text {inhom }}+3 H\left(\rho_{\text {inhom }}+p_{\text {inhom }}\right)=-q_{\varphi} \dot{\varphi}=-\frac{\beta}{M} \rho_{\text {inhom }} \sqrt{\rho_{h}+p_{h}}
\end{aligned}
$$

It is proportional to $\beta$.

(ii) The expansion of the universe accelerates if

$$
\frac{\ddot{a}}{a}=\dot{H}+H^{2}=-\frac{1}{12 M^{2}}\left(\rho_{t o t}+3 p_{t o t}\right)>0
$$

which is equivalent to a total equation of state $w_{t o t}=p_{t o t} / \rho_{t o t}<-1 / 3$. In terms of the equation of state for homogenous and inhomogenous quintessence $p_{h}=w_{h} \rho_{h}, p_{\text {inhom }}=$ $w_{\text {inhom }} \rho_{\text {inhom }}$ we can express

$$
w_{t o t}=w_{h} \Omega_{h}+w_{\text {inhom }}\left(1-\Omega_{h}\right)=w_{h} \Omega_{h}+w_{c} \Omega_{c}+w_{g} \Omega_{g}
$$


(iii) If gradient terms dominate over the time derivatives the cosmon equation of state is negative, $w_{c} \leq-1 / 3$ [15]. This may, however, be (partially) compensated by $w_{g} \Omega_{g}>0$. (iv) For fixed $\beta$ and $w_{h}$ we may write

$$
\dot{\rho}_{\text {inhom }}+n_{\text {inhom }}^{\text {eff }} H \rho_{\text {inhom }}=0, \quad n_{\text {inhom }}^{\text {eff }}=3\left(1+w_{\text {inhom }}\right)+\beta \sqrt{6 \Omega_{h}\left(1+w_{h}\right)}
$$

In case of negative $\beta$ and $w_{\text {inhom }}$ one finds $n_{\text {inhom }}^{\text {eff }}<3$. This would imply that the cosmon energy density dilutes shower than non-relativistic matter and, a fortiori, much slower than radiation.

(v) A decrease of $\beta$ towards negative values slows down the decrease of $\varphi$ (cf. eq. (2.12) with $\left.q_{\varphi}<0\right)$. This effect reduces the kinetic energy of the background field as compared to its potential energy and therefore drives $w_{h}$ towards negative values. Details depend on the form of the cosmon potential.

(vi) The relative change in homogenous quintessence obeys

$$
\dot{\Omega}_{h}=H\left(1-\Omega_{h}\right)\left\{3 \Omega_{h}\left(w_{\text {inhom }}-w_{h}\right)-\beta \sqrt{6\left(1+w_{h}\right)}\right\}
$$

Negative $\beta$ and negative $w_{h}$ tend to increase $\Omega_{h}$, whereas negative $w_{\text {inhom }}$ has the opposite effect.

(vii) It is conceivable that a change in the effective value of $\beta$ and $\omega_{\text {inhom }}$ after structure formation trigger an increase of $\Omega_{h}$ and an accelerated expansion of the universe [15].

We postpone a more detailed discussion of the interesting cosmon-dominated universe to a future investigation. Here we hope that we have motivated that such an investigation is worthwhile. We should be ready to encounter surprising effects as compared to standard cold dark matter cosmology with homogenous quintessence: The equation of state of cosmon dark matter may neither be the one of a relativistic nor of a nonrelativistic gas. It could even change with time. Homogenous quintessence has most likely a substantial coupling to cosmon dark matter, even though its coupling to ordinary matter vanishes or is very small. This introduces features that are not familiar in the standard hydrodynamical treatment of dark matter. In particular, the conservation equation (2.10) contains a modification $\sim q_{\varphi}$.

In order to get some insight into the effects of cosmon dark matter, for example by numerical simulations, one may first treat $\beta$ and $w_{\text {inhom }}$ as free parameters. Even though this is only a parametrization of our lack of knowledge we may learn how structure formation and the cosmic microwave background (CMB) are modified as compared to a standard cold dark matter inhomogenous component (for which $\beta=w_{\text {inhom }}=0$ ). We note that for cosmon dark matter $w_{\text {inhom }}$ could even be negative [15].

We also note that large cosmon fluctuations are not Gaussian any more, despite the likely approximate Gaussian distribution in early cosmology when the fluctuations were in the linear regime. The effect of the non-Gaussian behavior on the time evolution of the cosmon fluctuations will be briefly addressed in the next section. We recall, however, 
that even strong non-Gaussian cosmon fluctuations are compatible with approximately Gaussian fluctuations in the cosmon energy density and the CMB.

\section{Large fluctuations in mean field approximation}

In a cosmon-dominated universe the cosmon fluctuations are not small anymore and linear analysis does not apply. One may encounter gravitationally bound cosmon lumps [19] on all length scales. Extended cosmon lumps may become the seeds for galaxies and could describe the observed galactic halos. In this section we make a modest attempt to get a glance on the modifications of the cosmon fluctuation equations which are induced by the nonlinearities. We study a mean field approximation. This takes into account part of the effects of nonlinearities, while keeping a structure of the fluctuation equations similar to the linear analysis. In particular, the effective potential and the effective cosmon mass will depend on the amplitude of the fluctuations. Typically, the mass increases for the nonlinear system such that the inverse mass (correlation length) may become substantially smaller than the horizon. We notice, however, that the mean field analysis will fail to give a reliable description once gravitationally bound cosmon lumps form.

As a general framework for the analysis of nonlinear effects we may use the timedependent effective action [21]. For simplicity we neglect here the gravitational effects. This is only justified if nonlinearities occur also at scales somewhat smaller than the horizon. Since this is indeed the tendency of the mean field approximation we can investigate such a scenario in a self-consistent way. We start with the microscopic equation for the cosmon fluctuation

$$
\delta \ddot{\chi}_{k}+3 H \delta \dot{\chi}_{k}+\frac{k^{2}}{a^{2}} \delta \chi_{k}=f_{k}[\delta \chi]=-\int d^{3} x e^{-i k x}\left(V^{\prime}(\varphi+\delta \chi)-V^{\prime}(\varphi)\right)
$$

The quantity $f_{k}$ depends on the details of the potential. For our example of an exponential potential (6.1) $f_{k}$ can be represented by a Taylor series

$$
f_{k}=\frac{\alpha}{M} V(\varphi) \int d^{3} x e^{-i k x} \sum_{n=1}^{\infty} \frac{1}{n !}\left(-\frac{\alpha}{M} \delta \chi(x)\right)^{n}
$$

We introduce $\delta \pi_{k}=\delta \dot{\chi}_{k}$ and write eq. (10.1) as a coupled system of first-order differential equations (with $\delta \chi_{k}^{n}$ the Fourier transform of $\delta \chi^{n}(x)$ )

$$
\begin{aligned}
\delta \dot{\chi}_{k} & =\delta \pi_{k} \\
\delta \dot{\pi}_{k} & =-3 H \delta \pi_{k}-\frac{k^{2}}{a^{2}} \delta \chi_{k}+\frac{\alpha}{M} V(\varphi) \sum_{n=1}^{\infty} \frac{1}{n !}\left(-\frac{\alpha}{M} \delta \chi\right)_{k}^{n}
\end{aligned}
$$

For a given time evolution of the background quantities $a(t), H(t)$ and $\varphi(t)$ this is a closed system of "microscopic" equations. The time evolution of correlation functions for such 
systems can be described in terms of an exact evolution equation for a time-dependent effective action [21. We will be satisfied here with a simple "mean field" or "Hartree"-type approximation. We approximate

$$
\delta \chi_{k}^{n}=n<\delta \chi^{n-1}>\delta \chi_{k}
$$

with $<\delta \chi>=0$ and ( $m$ integer, $m \geq 2$ )

$$
\begin{aligned}
& <\chi^{2 m-1}>=0 \\
& <\chi^{2 m}>=3 \cdot 5 \ldots(2 m-3)(2 m-1)\left(<\chi^{2}>\right)^{m}
\end{aligned}
$$

The expectation value of the squared fluctuations is given again by eq. (3.2), $<\delta \chi^{2}>=A$. We note that $A$ receives contributions from all momentum modes, both larger and smaller than $k$. Already in the mean field approximation the evolution of $\delta \chi_{k}$ is therefore coupled to all other momentum modes.

In terms of $\alpha_{k}, \beta_{k}$ (cf. eq. (3.3)) and $\gamma_{k}$ (cf. eq. (3.12)) one obtains a closed system for the Fourier components of the two-point correlation

$$
\begin{aligned}
& \dot{\alpha}_{k} \equiv \beta_{\alpha_{k}}=2 \gamma_{k} \\
& \dot{\gamma}_{k} \equiv \beta_{\gamma_{k}}=\beta_{k}-3 H \gamma_{k}-\left(\frac{k^{2}}{a^{2}}+\mu^{2}\right) \alpha_{k} \\
& \dot{\beta}_{k} \equiv \beta_{\beta_{k}}=-6 H \beta_{k}-2\left(\frac{k^{2}}{a^{2}}+\mu^{2}\right) \gamma_{k}
\end{aligned}
$$

From eq. (10.6) we can derive the useful identity

$$
\partial_{t}\left(\alpha_{k} \beta_{k}-\gamma_{k}^{2}\right)=-6 H\left(\alpha_{k} \beta_{k}-\gamma_{k}^{2}\right)
$$

One infers a separate conservation law for every momentum mode

$$
a^{6}\left(\left(\alpha_{k} \beta_{k}-\gamma_{k}^{2}\right)=\right.\text { const }
$$

A modified effective mass term $\mu^{2}$ governs the cosmon fluctuations

$$
\mu^{2}=\frac{\alpha^{2}}{M^{2}} V\left(1+\sum_{m=1}^{\infty} g_{m}\left(\frac{\alpha^{2} A}{M^{2}}\right)^{m}\right)=\frac{\alpha^{2}}{M^{2}} V \mathcal{G}\left(\frac{\alpha^{2} A}{M^{2}}\right)
$$

with

$$
g_{m}^{-1}=2 \cdot 4 \cdot 6 \cdot 8 \cdots(2 m-2)(2 m)
$$

The new ingredient as compared to the linear approximation is the function $\mathcal{G}\left(\frac{\alpha^{2} A}{M^{2}}\right)$ which is equal to one for small fluctuations. We conclude that the mean field approximation leads to a time-dependent mass term which depends itself on the size of the fluctuations. 
The function $\mathcal{G}$ also appears in the nonlinear expressions for $\rho_{c}, p_{c}$ and $q_{\varphi}$

$$
\begin{aligned}
& \rho_{c}=V(\varphi)\left[\mathcal{G}\left(\frac{\alpha^{2} A}{M^{2}}\right)-1\right]+\frac{1}{2} B+\frac{C}{2 a^{2}} \\
& p_{c}=-V(\varphi)\left[\mathcal{G}\left(\frac{\alpha^{2} A}{M^{2}}\right)-1\right]+\frac{1}{2} B-\frac{C}{6 a^{2}} \\
& q_{\varphi}=\frac{\alpha}{M} V(\varphi)\left[\mathcal{G}\left(\frac{\alpha^{2} A}{M^{2}}\right)-1\right]
\end{aligned}
$$

and we may derive the relations

$$
\begin{gathered}
\mathcal{G}\left(\frac{\alpha^{2} A}{M^{2}}\right)=1+\frac{2 B-\rho-3 p}{2 V(\varphi)} \\
\mu^{2}=\frac{\alpha^{2}}{M^{2}}\left(V+B-\frac{\rho}{2}-\frac{3 p}{2}\right)
\end{gathered}
$$

Comparison with the linearized expressions (3.1) shows that the nonlinearities result essentially in a substitution $V^{\prime \prime}(\varphi) A / 2 \rightarrow \mu^{2} M^{2} / \alpha^{2}-V$, or

$$
\begin{aligned}
& \rho_{c}=\frac{\mu^{2} M^{2}}{\alpha^{2}}-V+\frac{1}{2} B+\frac{C}{2 a^{2}} \\
& p_{c}=-\left(\frac{\mu^{2} M^{2}}{\alpha^{2}}-V\right)+\frac{1}{2} B-\frac{C}{6 a^{2}} \\
& q_{\varphi}=\frac{\alpha}{M}\left(\frac{\mu^{2} M^{2}}{\alpha^{2}}-V\right)
\end{aligned}
$$

One may define a further effective mass term (different from $\mu^{2}$ !)

$$
m_{\rho}^{2}=2 V(\mathcal{G}-1) / A=\frac{2}{A}\left(\frac{\mu^{2} M^{2}}{\alpha^{2}}-V\right)=\frac{2 M^{2}}{\alpha^{2} A} \frac{\mathcal{G}-1}{\mathcal{G}} \mu^{2}
$$

which replaces $V^{\prime \prime}$ for the scalar contribution to $\rho_{c}$ etc. The nonlinear effects are reflected in the difference between $\mu^{2}, m_{\rho}^{2}$ and $V^{\prime \prime}(\varphi)$,

$$
\begin{aligned}
& \frac{\mu^{2}-V^{\prime \prime}(\varphi)}{V^{\prime \prime}(\varphi)}=\sum_{m=1}^{\infty} g_{m}\left(\frac{\alpha^{2} A}{M^{2}}\right)^{m}, \\
& \frac{m_{\rho}^{2}-V^{\prime \prime}(\varphi)}{V^{\prime \prime}(\varphi)}=\sum_{m=1}^{\infty} 2 g_{m+1}\left(\frac{\alpha^{2} A}{M^{2}}\right)^{m}
\end{aligned}
$$

We conclude that the discussion of the cosmon fluctuations in the mean field approximation parallels the linear approximation in many aspects, with two important modifications: 
(a) the mass term $\mu^{2}$ replaces $V^{\prime \prime}$ in eqs. (3.6), (3.7), 3.10), (3.11), (3.13),

(b) the relation (3.1) and similar relations for $\rho_{k}$ and $p_{k}$ get modified by replacing $V^{\prime \prime}$ by $m_{\rho}^{2}$.

Let us first consider the short distance modes with $k^{2} / a^{2} \gg \mu^{2}, H^{2}$. We assume that $A$ varies only slowly on the time scale of the corresponding oscillation and neglect $\dot{A}$ in a first approximation. One therefore finds again in leading order that particle production is suppressed, $\Delta_{k}=0$. The relation between $\rho_{k}, p_{k}$ and $n_{k}$ reads now (for $\Delta_{k}=0$ and the definition of $n_{k}$ involving $\mu^{2}$ instead of $V^{\prime \prime}$ )

$$
\begin{aligned}
& \rho_{k}=\frac{1}{2}\left\{\left(\frac{k^{2}}{a^{2}}+m_{\rho}^{2}\right) \alpha_{k}+\beta_{k}\right\}=\left(\frac{k^{2}}{a^{2}}+\mu^{2}\right)^{1 / 2} n_{k}+\frac{1}{2} \Delta m^{2} \alpha_{k} \\
& p_{k}=\frac{1}{2}\left\{\beta_{k}-\left(m_{\rho}^{2}+\frac{k^{2}}{3 a^{2}}\right) \alpha_{k}\right\}=\frac{k^{2}}{3 a^{2}}\left(\frac{k^{2}}{a^{2}}+\mu^{2}\right)^{-1 / 2} n_{k}-\frac{1}{2} \Delta m^{2} \alpha_{k}
\end{aligned}
$$

Beyond the replacement of $V^{\prime \prime}$ by $\mu^{2}$ we observe additional terms involving the difference of the two types of effective masses

$$
\Delta m^{2}=m_{\rho}^{2}-\mu^{2}=-V^{\prime \prime} \sum_{m=1}^{\infty} \frac{m}{m+1} g_{m}\left(\frac{\alpha^{2} A}{M^{2}}\right)^{m}
$$

For large enough $k^{2} / a^{2}$ this new effect is suppressed, however, yielding a typical relative correction to the equation of state $\sim \Delta m^{2} a^{2} / k^{2}$. We conclude that in the mean field approximation the high momentum modes again behave as relativistic particles. If they would dominate, the equation of state would be $p \approx \rho / 3$. Neglecting the time evolution of $a, V$ and $A$, the deviation from the relativistic behavior due to masslike terms is governed by

$$
\frac{p_{k}}{\rho_{k}}=\frac{1}{3}\left[1+\frac{a^{2}}{k^{2}}\left(\mu^{2}-2 m_{\rho}^{2}\right)+0\left(\left(\frac{m^{2} a^{2}}{k^{2}}\right)^{2}\right)\right]
$$

In lowest order in $\alpha^{2} A / M^{2}$ the nonlinear effects cancel for this relation, $\mu^{2}=-2 m_{\rho}^{2}=$ $-V^{\prime \prime}\left(1+0\left(\left(\alpha^{2} A / M^{2}\right)^{2}\right)\right.$.

We conclude that in the mean field approximation the short distance modes $\left(k^{2} / a^{2} \gg\right.$ $\left.\mu^{2}\right)$ behave as relativistic matter even in presence of nonlinear effects. In this approximation the interesting candidates for cosmon dark matter are therefore fluctuations with $k^{2} / a^{2}$ comparable to $\mu^{2}, m_{\rho}^{2}$ or smaller. An interesting situation would arise if these fluctuations have a wavelength well within the horizon, which requires $\mu^{2} \gg H^{2}$ or $m_{\rho}^{2} \gg H^{2}$. In order to see if this is possible, we first note the inequalities

$$
\rho_{c} \geq \frac{1}{2} m_{\rho}^{2} A, \quad H^{2} \geq \frac{1}{12} \frac{m_{\rho}^{2} A}{M^{2}}
$$


On the other hand, nonlinear effects are important only for $\frac{A}{M^{2}} \geq \frac{1}{\alpha^{2}}$, and we conclude for the nonlinear regime

$$
\frac{m_{\rho}^{2}}{H^{2}} \leq 12 \alpha^{2}
$$

For $\alpha$ sufficiently large $m_{\rho}$ could indeed be substantially larger than $H$. Furthermore, for large $x=\alpha^{2} A / M^{2}$ the ratio $\mu^{2} / m_{\rho}^{2} \approx x / 2$ is also large.

\section{Conclusions}

In conclusion, the cosmon dark matter scenario seems plausible enough to merit a detailed investigation. Several important questions have been left open in the present paper: a precise treatment of the time evolution of fluctuations with wavelength around the horizon, a quantitative investigation of a possible cosmon-dominated epoch, a discussion of the time evolution of fluctuations in the radiation (relevant for the CMB), a description of structure formation, the role of possible gravitationally bound cosmon lumps, the influence of structure formation on the time evolution of quintessence... The present note presents only the basic ideas and first arguments in favor of a cosmon dark matter scenario. In particular, we have discussed a setting where the energy density of the cosmon fluctuations grows relative to radiation during a radiation-dominated period. Our paper also develops a general theoretical framework to investigate the relevant questions in a context where nonlinearities and backreaction effects become important. Much remains to be done, however, before a consistent "cosmon dark matter cosmology" is developed.

Nevertheless, two important general conclusions can be drawn for a possible cosmon dark matter scenario. (1) An unusual equation of state may arise not only for the homogenous quintessence component but also for the part of dark matter taking part in structure formation. The effective equation of state may depend on time and perhaps even on the particular problem. It is conceivable that the effective equation of state differs for extended spherically symmetric objects (e. g. cosmon lumps) and for a homogenous cosmology. (2) One expects a sizeable coupling of quintessence to cosmon dark matter. This modifies the energy momentum conservation for dark matter and influences structure formation as well as the details of the cosmic microwave background.

Most important, the cosmon dark matter scenario opens the perspective of a unified description of homogenous dark energy and "clumping" dark matter. It has the potential to explain the rough equality of these two components as well as to answer the question of "why now" for the present acceleration, without putting in the answers "by hand" in the form of appropriately tuned potentials or kinetic terms. A success of these ideas would greatly enhance the naturalness of quintessence. For experimental dark matter searches the consequences would be dramatic: cosmons interact only with gravitational strength and cannot be detected by present and planned laboratory experiments. No new dark 
matter particle would be needed. In the forseeable future dark matter would show up only in an astrophysical or cosmological context!

\section{Acknowledgement}

The author would like to thank C. M. Müller for pointing out an omission in a previous formulation of appendix A.

\section{Appendix A}

\section{Evolution equation for metric fluctuations}

In this appendix we derive the microscopic field equation for the fluctuations of the metric, $h_{\mu \nu}=g_{\mu \nu}-\bar{g}_{\mu \nu}$, in linear order. We concentrate on a Robertson-Walker (zero curvature) background $\bar{g}_{\mu \nu}$ and the harmonic background gauge $D_{\mu} h^{\mu}{ }_{\nu}=\frac{1}{2} \partial_{\nu} h$. The starting point is the Einstein equation

$$
R_{\mu \nu}-\frac{1}{2} R g_{\mu \nu}=\frac{1}{2 M^{2}} t_{\mu \nu}
$$

The linear expansion for the Fourier modes with comoving momenta $k \neq 0$ yields

$$
\left(\delta R_{\mu \nu}\right)_{k}=\frac{1}{2 M^{2}}\left(\delta s_{\mu \nu}\right)_{k}
$$

with

$$
\begin{aligned}
& s_{\mu \nu}=t_{\mu \nu}-\frac{1}{2} t_{\rho}^{\rho} g_{\mu \nu}, \quad \delta s_{\mu \nu}=s_{\mu \nu}-\bar{s}_{\mu \nu} \\
& \bar{s}_{\mu \nu}=\hat{T}_{\mu \nu}-\frac{1}{2} \hat{T}_{\rho \sigma} \bar{g}^{\rho \sigma} \bar{g}_{\mu \nu}
\end{aligned}
$$

We observe that we have to subtract the background energy momentum tensor which includes fluctuation effects that are already taken into account in the determination of the background solution. Therefore $\delta t_{\mu \nu}=t_{\mu \nu}-\hat{T}_{\mu \nu}$ is the microscopic energy tensor for the cosmon and metric fluctuations around the background solution. Here

$$
\hat{T}_{\mu \nu}=T_{\mu \nu}^{\varphi}+T_{\mu \nu} \quad, \quad T_{\mu \nu}^{\varphi}=\partial_{\mu} \varphi \partial_{\nu} \varphi+\left(\frac{1}{2} \dot{\varphi}^{2}-V\right) \bar{g}_{\mu \nu}
$$

includes the incoherent effects in $T_{\mu \nu}$. As a result, the homogenous part ( $k=0$ component) has always to be subtracted. Defining $V(\varphi+\delta \chi)=V(\varphi)+\delta V$ one has, for example, the fluctuation contribution

$$
(V(\varphi+\delta \chi)-<V>)_{k}=\delta V_{k}-\delta V_{k=0}
$$


We first need the expansion of the Ricci tensor

$$
\begin{aligned}
\left(\delta R_{\mu \nu}\right)_{k}= & \left(\partial_{\lambda} \delta \Gamma_{\mu \nu}^{\lambda}\right)_{k}-\left(\partial_{\mu} \delta \Gamma_{\lambda \nu}{ }^{\lambda}\right)_{k} \\
& +\Gamma_{\mu \nu}{ }^{\tau}\left(\delta \Gamma_{\lambda \tau}^{\lambda}\right)_{k}+\Gamma_{\lambda \tau}{ }^{\lambda}\left(\delta \Gamma_{\mu \nu}^{\tau}\right)_{k}-\Gamma_{\lambda \nu}{ }^{\tau}\left(\delta \Gamma_{\mu \tau}^{\lambda}\right)_{k}-\Gamma_{\mu \tau}{ }^{\lambda}\left(\delta \Gamma_{\lambda \nu}{ }^{\tau}\right)_{k}
\end{aligned}
$$

Here $\Gamma_{\mu \nu}^{\lambda}$ is the connection for the homogenous and isotropic background metric and

$$
\begin{aligned}
\left(\delta \Gamma_{\mu \nu}^{\lambda}\right)_{k} & =\frac{1}{2} \bar{g}^{\lambda \rho}\left(\partial_{\mu} h_{\nu \rho}+\partial_{\nu} h_{\mu \rho}-\partial_{\rho} h_{\mu \nu}\right)_{k}-\Gamma_{\mu \nu}^{\tau}\left(h_{\tau}^{\lambda}\right)_{k} \\
& =\frac{1}{2}\left(h_{\nu ; \mu}^{\lambda}+h_{\mu ; \nu}^{\lambda}-h_{\mu \nu ;}^{\lambda}\right)
\end{aligned}
$$

We omit in the following the bar for the background metric which is used to raise and lower the indices of $h_{\mu \nu}$ and also the momentum label $k$ for $h_{\mu \nu}$. One obtains $\left(h=h_{\lambda}{ }^{\lambda}\right)$

$$
\begin{aligned}
\delta R_{\mu \nu} & =\left(\delta \Gamma_{\mu \nu}^{\lambda}\right)_{; \lambda}-\left(\delta \Gamma_{\lambda \nu}^{\lambda}\right)_{; \mu} \\
& =\frac{1}{2}\left(h_{\mu ; \nu \lambda}^{\lambda}+h_{\nu ; \mu \lambda}^{\lambda}-h_{\mu \nu ; \lambda}^{\lambda}-h_{; \nu \mu}\right)
\end{aligned}
$$

We note that with respect to gauge transformations of the background metric $h_{\mu \nu}$ transforms as a tensor. Using the identity

$$
h_{\mu ; \nu \tau}^{\lambda}-h_{\mu ; \tau \nu}^{\lambda}=R_{\nu \tau \mu}^{\sigma} h_{\sigma}^{\lambda}-R_{\nu \tau \sigma}^{\lambda} h_{\mu}^{\sigma}
$$

this yields

$$
\delta R_{\mu \nu}=-\frac{1}{2} h_{\mu \nu ; \lambda}^{\lambda}-R_{\mu \lambda \nu \sigma} h^{\lambda \sigma}+\frac{1}{2} R_{\nu \sigma} h_{\mu}^{\sigma}+\frac{1}{2} R_{\mu \sigma} h_{\nu}^{\sigma}+\delta K_{\mu \nu}
$$

with

$$
\delta K_{\mu \nu}=\frac{1}{2}\left(h_{\mu ; \lambda \nu}^{\lambda}-\frac{1}{2} h_{; \mu \nu}+h_{\nu ; \lambda \mu}^{\lambda}-\frac{1}{2} h_{; \nu \mu}\right)
$$

The harmonic background gauge implies $\delta K_{\mu \nu}=0$. In particular, one finds for the trace

$$
g^{\mu \nu} \delta R_{\mu \nu}=-\frac{1}{2} h_{; \mu}^{\mu}
$$

which yields the field equation for the trace of the metric fluctuations in an arbitrary background

$$
\begin{aligned}
h_{; \mu}^{\mu} & =-\frac{1}{M^{2}} \delta s_{\mu}^{\mu}=\frac{1}{M^{2}}\left(\delta t_{\mu}^{\mu}-2 \hat{T}^{\mu \nu} h_{\mu \nu}+\frac{1}{2} \hat{T}_{\rho}^{\rho} h_{\mu}^{\mu}\right) \\
& =\frac{1}{M^{2}}\left[\delta t_{\mu}^{\mu}-\frac{1}{2}\left(\dot{\varphi}^{2}+\rho+p\right)\left(h+4 h_{00}\right)\right]
\end{aligned}
$$


where $\delta t_{\mu}^{\mu}=\bar{g}^{\mu \nu} \delta t_{\mu \nu} \neq \delta\left(g^{\mu \nu} t_{\mu \nu}\right)$.

We also need an evolution equation for $h_{00}$, i.e.

$$
h_{00 ; \lambda}{ }_{\lambda}^{\lambda}+2 R_{0 \lambda 0 \sigma} h^{\lambda \sigma}-2 R_{0}{ }^{\sigma} h_{0 \sigma}=-\frac{1}{M^{2}} \delta s_{00}
$$

Inserting the connection for the Robertson-Walker metric, $\Gamma_{i j}{ }^{0}=H g_{i j}, \Gamma_{0 i}{ }^{j}=H \delta_{i}^{j}$, zero otherwise, the curvature tensor reads $R_{0 i 0}{ }^{j}=-\left(H^{2}+\dot{H}\right) \delta_{i}^{j}, R_{00}=-3\left(H^{2}+\dot{H}\right)$ such that

$$
h_{00 ; \lambda}^{\lambda}-2\left(H^{2}+\dot{H}\right)\left(h+4 h_{00}\right)=-\frac{1}{M^{2}} \delta s_{00}
$$

We finally employ the explicit expressions $h_{; \lambda}^{\lambda}=-\left(\ddot{h}+3 H \dot{h}+\left(k^{2} / a^{2}\right) h\right), h_{00 ; \lambda}{ }_{\lambda}^{\lambda}=-\left(\ddot{h}_{00}+\right.$ $\left.3 H \dot{h}_{00}+\left(k^{2} / a^{2}\right) h_{00}-2 H^{2}\left(h+4 h_{00}\right)+4 H \partial_{i} h_{0}^{i}\right)$ and the gauge condition

$$
\partial_{i} h_{0}^{i}=\frac{1}{2} \dot{h}+\dot{h}_{00}+H\left(h+4 h_{00}\right)
$$

and infer

$$
\begin{aligned}
& \ddot{h}+3 H \dot{h}+\frac{k^{2}}{a^{2}} h=\frac{1}{M^{2}} \delta s_{\mu}^{\mu} \\
& \ddot{h}_{00}+H\left(2 \dot{h}+7 \dot{h}_{00}\right)+2\left(2 H^{2}+\dot{H}\right)\left(h+4 h_{00}\right)+\frac{k^{2}}{a^{2}} h_{00}=\frac{1}{M^{2}} \delta s_{00}
\end{aligned}
$$

Using again the harmonic gauge condition

$$
\partial_{j} h_{i}^{j}=\frac{1}{2} \partial_{i} h+\dot{h}_{0 i}+3 H h_{0 i}
$$

we obtain similarly

$$
\ddot{h}_{0 i}+3 H \dot{h}_{0 i}+\frac{k^{2}}{a^{2}} h_{0 i}+\left(6 H^{2}+5 \dot{H}\right) h_{0 i}+H\left(\partial_{i} h+2 \partial_{i} h_{00}\right)=\frac{1}{M^{2}} \delta s_{0 i}
$$

For a solution of the metric field equation we need the source terms $\delta s_{\mu \nu}$. Defining $\delta t_{00}=\delta \rho, \delta t_{i j}=\delta p \bar{g}_{i j}$ one obtains

$\delta s_{00}=\frac{1}{2}\left[\delta \rho+3 \delta p-\left(p-V+\frac{1}{2} \dot{\varphi}^{2}\right)\left(h+4 h_{00}\right)\right], \bar{g}^{i j} \delta s_{i j}=\frac{3}{2}(\delta \rho-\delta p)+\frac{1}{2}\left(\rho+V+\frac{1}{2} \dot{\varphi}^{2}\right)\left(h+4 h_{00}\right)$

or

$$
\delta s_{\rho}^{\rho}=\delta \rho-3 \delta p+\frac{1}{2}\left(\dot{\varphi}^{2}+\rho+p\right)\left(h+4 h_{00}\right)
$$


For the cosmon the microscopic fluctuations $\delta \rho$ and $\delta p$ are linear in the fluctuations $\delta \chi, \delta \dot{\chi}$, in contrast to usual matter like baryons or photons where $\delta \rho$ and $\delta p$ are quadratic in the fluctuating field. One has

$$
\begin{aligned}
\delta \rho_{k} & =V^{\prime} \delta \chi_{k}+\dot{\varphi} \delta \dot{\chi}_{k}-V\left(h_{00}\right)_{k} \\
\delta p_{k} & =-V^{\prime} \delta \chi_{k}+\dot{\varphi} \delta \dot{\chi}_{k}-\frac{1}{3}\left(V-\frac{1}{2} \dot{\varphi}^{2}\right)\left(h+h_{00}\right)_{k}+\frac{1}{2} \dot{\varphi}^{2}\left(h_{00}\right)_{k}
\end{aligned}
$$

For eq. (A.20) we also need

$$
\delta s_{0 i}=\delta t_{0 i}-\frac{1}{2} \hat{T}_{\rho}^{\rho} h_{0 i}=\dot{\varphi} \partial_{i} \delta \chi+\left(V-\frac{1}{2} \rho+\frac{3}{2} p\right) h_{0 i}
$$

One observes that the rotation vector $h_{0 i}$ couples to the rotation scalars $h_{00}, h, \delta \chi$ only through the derivatives $\partial_{i} h, \partial_{i} h_{00}, \partial_{i} \delta \chi$ in eqs. (A.20), (A.24).

We are particularly interested in the evolution of the modes with length scale outside the horizon, $k^{2} / a^{2} \ll H^{2}$. For these modes the coupling between $h_{0 i}$ and $h, h_{00}, \delta \chi$ becomes suppressed and vanishes for $k^{2} \rightarrow 0$. A possible solution appears to be $h_{0 i} \sim$ $H^{-1} \partial_{i} h$ or $\left(h_{0 i}\right)_{k} \sim i k_{i} h / H$, since $\left(h_{0 i}\right)_{k}=0$ is indeed a solution of eq. (A.20) for $k^{2}=0$. For this type of solution an important technical simplification happens for the long-distance modes: as compared to $h$ the quantity

$$
H^{-1} \partial_{i} h_{0}{ }^{i} \sim H^{-2} \frac{k^{2}}{a^{2}} h
$$

is small and may be neglected. The gauge condition (A.16) simplifies

$$
\dot{h}+2 \dot{h}_{00}+2 H\left(h+4 h_{00}\right)=0
$$

and we do not need to consider $h_{0 i}$ further. The gauge condition (A.26) can then be used instead of the field equation (A.18) in order to determine the time evolution of $h_{00}$. In particular, for $h \sim t^{\lambda}, h_{00} \sim t^{\lambda}$, and $H=\eta t^{-1}$ one finds from (A.26)

$$
\lambda h+2 \lambda h_{00}+2 \eta\left(h+4 h_{00}\right)=0
$$

This yields the ratio

$$
y=h_{00} / h=-(\lambda+2 \eta) /(2 \lambda+8 \eta)
$$

We have investigated the self-consistency of a possible solution with $\partial_{i} h_{0}^{i} \approx 0$ only for the case $\lambda=0$ (see sect. 8). The question if a solution with the property (A.25) also exists for $\lambda>0$ still needs a more thorough discussion, in particular in presence of fluctuations in the radiation. 


\section{Appendix B}

\section{Gravitational contribution to the energy momentum tensor}

In this appendix we compute the gravitational contribution to the energy momentum tensor, eq. (2.9). We start be evaluating $R_{\mu \nu}$ in second order in $h_{\mu \nu}$. Using

$$
\begin{gathered}
g^{\mu \nu}=\bar{g}^{\mu \nu}-h^{\mu \nu}+h_{\rho}^{\mu} h^{\rho \nu} \\
\left.\Gamma_{\mu \nu}^{\lambda}=\bar{\Gamma}_{\mu \nu}^{\lambda}+\frac{1}{2}\left(\bar{g}^{\lambda \rho}-h^{\lambda \rho}\right) h_{\nu \rho ; \mu}+h_{\mu \rho ; \nu}-h_{\mu \nu ; \rho}\right)
\end{gathered}
$$

one finds

$$
\begin{aligned}
R_{\mu \nu}= & \bar{R}_{\mu \nu}+\frac{1}{2}\left[\left(\bar{g}^{\lambda \rho}-h^{\lambda \rho}\right)\left(h_{\nu \rho ; \mu}+h_{\mu \rho ; \nu}-h_{\mu \nu ; \rho}\right)\right]_{; \lambda} \\
& -\frac{1}{2}\left[\left(\bar{g}^{\lambda \rho}-h^{\lambda \rho}\right)\left(h_{\nu \rho ; \lambda}+h_{\lambda \rho ; \nu}-h_{\lambda \nu ; \rho}\right)\right]_{; \mu} \\
& +\frac{1}{4}\left\{h_{;}^{\rho}\left(h_{\mu \rho ; \nu}+h_{\nu \rho ; \mu}-h_{\mu \nu ; \rho}\right)\right. \\
& \left.-\left(h_{\mu ; \lambda}^{\rho}+h_{\lambda ; \mu}^{\rho}-h_{\mu \lambda ;}^{\rho}\right)\left(h_{\rho ; \nu}^{\lambda}+h_{\nu ; \rho}^{\lambda}-h_{\rho \nu ;}{ }^{\lambda}\right)\right\} \\
= & \bar{R}_{\mu \nu}+\frac{1}{2}\left(h_{\nu \rho ; \mu}^{\rho}+h_{\mu \rho ; \nu}^{\rho}-h_{\mu \nu ; \rho}^{\rho}-h_{; \nu \mu}\right) \\
& -\frac{1}{2} h^{\lambda \rho}\left(h_{\nu \rho ; \mu \lambda}+h_{\mu \rho ; \nu \lambda}-h_{\mu \nu ; \rho \lambda}-h_{\rho \lambda ; \nu \mu}\right) \\
& -\frac{1}{2}\left(h_{; \lambda}^{\rho \lambda}-\frac{1}{2} h_{;}^{\rho}\right)\left(h_{\nu \rho ; \mu}+h_{\mu \rho ; \nu}-h_{\mu \nu ; \rho}\right) \\
& +\frac{1}{4} h_{; \mu}^{\rho \lambda} h_{\lambda \rho ; \nu}-\frac{1}{2} h_{\mu ; \rho}^{\lambda} h_{\nu ; \lambda}^{\rho}+\frac{1}{2} h_{\mu \lambda ;}^{\rho} h_{\nu ; \rho}^{\lambda}
\end{aligned}
$$

In lowest order only the terms quadratic in $h_{\mu \nu}$ contribute to $<\delta G_{\mu \nu}>$ :

$$
\begin{aligned}
<\delta G_{\mu \nu}>= & <\delta R_{\mu \nu}-\frac{1}{2} \delta\left(R g_{\mu \nu}\right)> \\
= & <\delta^{(2)} R_{\mu \nu}-\frac{1}{2} \delta^{(2)} R_{\sigma \tau} \bar{g}^{\sigma \tau} \bar{g}_{\mu \nu}+\frac{1}{2} \delta^{(1)} R_{\sigma \tau}\left(h^{\sigma \tau} \bar{g}_{\mu \nu}-\bar{g}^{\sigma \tau} h_{\mu \nu}\right) \\
& -\frac{1}{2} \bar{R}_{\sigma \tau}\left(h_{\rho}^{\sigma} h^{\rho \tau} \bar{g}_{\mu \nu}-h^{\sigma \tau} h_{\mu \nu}\right)> \\
= & <-\frac{1}{2} h^{\lambda \rho}\left(h_{\nu \rho ; \mu \lambda}+h_{\mu \rho ; \nu \lambda}-h_{\mu \nu ; \rho \lambda}-h_{\rho \lambda ; \nu \mu}\right)-\frac{1}{2} h_{\mu \nu}\left(h_{; \lambda \rho}^{\lambda \rho}-h_{; \rho}^{\rho}\right) \\
& -\frac{1}{2}\left(h_{; \lambda}^{\rho \lambda}-\frac{1}{2} h_{;}^{\rho}\right)\left(h_{\nu \rho ; \mu}+h_{\mu \rho ; \nu}-h_{\mu \nu ; \rho}\right)
\end{aligned}
$$




$$
\begin{aligned}
& +\frac{1}{4} h_{; \mu}^{\rho \lambda} h_{\lambda \rho ; \nu}-\frac{1}{2} h_{\mu ; \rho}^{\lambda} h_{\nu ; \lambda}^{\rho}+\frac{1}{2} h_{\mu \lambda ;}{ }^{\rho} h_{\nu ; \rho}^{\lambda}+\frac{1}{2} \bar{R}_{\rho \lambda} h^{\rho \lambda} h_{\mu \nu}> \\
& +\bar{g}_{\mu \nu}<\frac{1}{2} h^{\lambda \rho}\left(h_{\sigma \rho ; \lambda}^{\sigma}+h_{\sigma \rho ; \lambda}{ }^{\sigma}-h_{; \rho \lambda}-h_{\rho \lambda ; \sigma}{ }^{\sigma}\right) \\
& +\frac{1}{2}\left(h_{; \lambda}^{\rho \lambda}-\frac{1}{2} h_{;}{ }^{\rho}\right)\left(h_{\rho ; \sigma}^{\sigma}-\frac{1}{2} h_{; \rho}\right)+\frac{1}{4} h_{\sigma ; \rho}{ }^{\lambda} h_{; \lambda}^{\sigma \rho}-\frac{3}{8} h_{\sigma \lambda ;}{ }^{\rho} h_{; \rho}^{\sigma \lambda}>
\end{aligned}
$$

This expression can be somewhat simplified by use of the harmonic gauge condition

$$
\begin{aligned}
\delta G_{\mu \nu}= & \frac{1}{2}<h^{\lambda \rho}\left(h_{\mu \nu ; \rho \lambda}+h_{\rho \lambda ; \mu \nu}-h_{\nu \rho ; \mu \lambda}-h_{\mu \rho ; \nu \lambda}\right) \\
& +\frac{1}{2} h_{\mu \nu} h_{; \rho}{ }^{\rho}+\frac{1}{2} h_{; \mu}^{\rho \lambda} h_{\lambda \rho ; \nu}-h_{\mu ; \rho}^{\lambda} h_{\nu ; \lambda}^{\rho}+h_{\mu \lambda ;}{ }^{\rho} h_{\nu ; \rho}^{\lambda}+\bar{R}_{\rho \lambda} h^{\rho \lambda} h_{\mu \nu}> \\
& -\frac{1}{2} \bar{g}_{\mu \nu}<h^{\lambda \rho} h_{\rho \lambda ; \sigma}{ }^{\sigma}-\frac{1}{2} h_{\sigma ; \rho}^{\lambda} h_{; \lambda}^{\sigma \rho}+\frac{3}{4} h_{\sigma \lambda ;}{ }^{\rho} h_{; \rho}^{\sigma \lambda}-h^{\lambda \rho}\left(\bar{R}_{\lambda \tau} h_{\rho}^{\tau}-\bar{R}_{\lambda \sigma \rho \tau} h^{\sigma \tau}\right)>
\end{aligned}
$$

Eq. (2.9) yields the gravitational energy density $\rho_{g}$ and pressure $p_{g}$.

We are interested here in the contribution from fluctuations with wavelength larger than the horizon哓. We therefore can neglect the space derivatives and approximate $h_{0 i}=0$. Inserting the Robertson-Walker background one finds

$$
\begin{aligned}
\delta G_{\mu \nu}= & \frac{1}{2} \bar{g}_{\mu \nu}<\frac{1}{3} h\left(\ddot{h}+\ddot{h}_{00}\right)+\frac{1}{3} h_{00}\left(\ddot{h}+4 \ddot{h}_{00}\right)+\frac{1}{4} \dot{h}_{00}^{2}+\frac{3}{4} \dot{h}_{i}^{j} \dot{h}_{j}^{i} \\
& +H\left\{\frac{5}{3} h \dot{h}+\frac{7}{3} h \dot{h}_{00}+\frac{14}{3} h_{00} \dot{h}+\frac{31}{3} h_{00} \dot{h}_{00}+\frac{1}{2} h_{j}^{i} h_{j}^{i}\right\} \\
& +H^{2}\left\{-\frac{1}{3} h^{2}+\frac{19}{3} h h_{00}+\frac{103}{6} h_{00}^{2}+\frac{19}{4} h_{i}^{j} h_{j}^{i}\right\} \\
& +\dot{H}\left\{2 h h_{00}+5 h_{00}^{2}+h_{i}^{j} h_{j}^{i}\right\}>+\delta F_{\mu \nu}
\end{aligned}
$$

with $\delta F_{\mu \nu}$ a similar contribution from the first bracket in eq. (B.5). For the constant solution, $h_{00}=-\frac{1}{4} h, \dot{h}=0$, a radiation background $\dot{H}=-2 H^{2}$, and using $h_{i j}=$ $\frac{1}{3} h_{i}{ }^{i} g_{i j}=\frac{1}{3}\left(h+h_{00}\right) g_{i j}$ one obtains

$$
\left(\rho_{g}\right)_{k}=\frac{c}{8} M^{2} H^{2}<h^{2}>_{k}=\frac{c \alpha^{2}}{2 t^{2}} \alpha_{k}
$$

\section{Appendix $\mathrm{C}$}

\section{Naming scheme for energy densities}

In this appendix we summarize our naming scheme for the different contributions to the energy density $\rho_{j}$, and similar for $p_{j}$ and $\Omega_{j}$ :

\footnotetext{
${ }^{15}$ See $[15$ for a discussion of the wavelengths inside the horizon.

${ }^{16}$ We have not yet computed the proportionality factor $c$ which could vanish.
} 
$\rho_{h}$ : homogenous quintessence; smoothly distributed over distances $z \leq 10$.

$\rho_{c}$ : cosmon; contribution of scalar fluctuations with wavelength $z \leq 10$.

$\rho_{\varphi}:$ scalar; $\rho_{\varphi}=\rho_{h}+\rho_{c}$

$\rho_{g}:$ gravitational

$\rho_{q}:$ quintessence; $\rho_{q}=\rho_{\varphi}+\rho_{g}$

$\lambda$ : cosmological constant $\left(\rho_{\Lambda} \equiv \lambda\right)$

$\rho_{d e}:$ dark energy; $\rho_{d e}=\rho_{h}+\lambda$

$\rho_{c d m}:$ cold dark matter

$\rho_{d m}:$ dark matter; $\rho_{d m}=\rho_{c}+\rho_{g}+\rho_{c d m}\left(+\rho_{\nu}+\ldots\right)$

$\rho_{d}:$ dark; $\rho_{d}=\rho_{d e}+\rho_{d m}$.

$\rho_{b}$ : baryons; $\quad \rho_{\nu}$ : neutrinos; $\quad \rho_{r}$ : radiation

$\rho_{m}:$ nonrelativistic matter; $\rho_{m}=\rho_{c d m}+\rho_{b}\left(+\rho_{\nu}+\ldots\right)$

$\rho_{p}:$ particles; $\rho_{p}=\rho_{c d m}+\rho_{r}+\rho_{\nu}+\rho_{b}+\ldots$

$\rho_{\text {inhom }}$ : inhomogenous; takes part in structure formation; $\rho_{\text {inhom }}=\rho_{c}+\rho_{g}+\rho_{m}$ (we often use $\rho_{\text {inhom }} \rightarrow \rho$ )

$\rho_{\text {tot }}:$ total; $\rho_{\text {tot }}=\rho_{\text {de }}+\rho_{\text {inhom }}+\rho_{r}+\ldots$

An interesting candidate for recent and present cosmology would be a universe consisting mainly of homogenous quintessence and cosmon dark matter, $\rho_{t o t}=\rho_{h}+\rho_{c}+\rho_{g}=\rho_{q}=$ $\rho_{h}+\rho_{\text {inhom }}$.

\section{References}

[1] C. Wetterich, Nucl. Phys. B302 (1988) 668

[2] P. J. E. Peebles and B. Ratra, Astrophys. Lett. 325 (1988) L17;

B. Ratra and P. J. E. Peebles, Phys. Rev. D37 (1988) 3406

[3] E. Copeland, A. Liddle, D. Wands, Ann. N.Y. Acad. Sci 688 (1993) 647;

Phys. Rev. D57 (1998) 4686 
[4] C. Wetterich, Astron. Astrophys. 301 (1995) 321

[5] M. Reuter, C. Wetterich, Phys. Lett. B188 (1987) 38

[6] C. Wetterich, Nucl. Phys. B302 (1988) 645

[7] R. Caldwell, R. Dave, and P. Steinhardt, Phys. Rev. Lett. 80 (1998) 1582;

P. Steinhardt, L. Wang, and I. Zlatev, Phys. Rev. D59 (1999) 123504

[8] P. G. Ferreira and M. Joyce, Phys. Rev. D58 (1998) 023503

[9] C. B. Netterfield et al., astro-ph/0104460

[10] A. G. Riess et al., Astron. J. 116 (1998) 1009;

S. Perlmutter et al., Astrophys. J. 517 (1999) 565

[11] R. Bean, S. Hansen, and A. Melchiorri, Phys. Rev. D64 (2001) 103508;

M. Doran, M. Lilley, and C. Wetterich, astro-ph/0105457;

P. Corasaniti and E. Copeland, Phys. Rev. D65 (2002) 043004;

M. Doran, M. Schwindt, and C. Wetterich, Phys. Rev. D64 (2001) 123520;

C. Baccigalupi, A. Balbi, S. Matarrese F. Perrotta, and N. Vittorio, astro-ph/0109097;

R. Bean, A. Melchiorri, Phys. Rev. D65 (2002) 041302.

[12] A. Albrecht and C. Skordis, Phys. Rev. Lett. 84 (2000) 2076

[13] V. Sahni and L. Wang, Phys. Rev. D62 (2000) 103517

[14] A. Hebecker and C. Wetterich, Phys. Lett. B497 (2001) 281

[15] C. Wetterich, astro-ph/0111166

[16] T. Ott, Phys. Rev. D64 (2001) 023518

[17] L. Amendola, Phys. Rev. D62 (2000) 043511

[18] C. Amendariz-Picon, V. Mukhanov, and P. J. Steinhardt, Phys. Rev. Lett. 85 (2000) 4438; Phys. Rev. D63 (2001) 103510

[19] C. Wetterich, Phys. Lett. B522 (2001) 5

[20] R. Brandenberger, hep-th/0004016; M. Li, W. Lin, X. Zhang, and R. Brandenberger, Phys. Rev. D65 (2002) 023519

[21] C. Wetterich, Phys. Rev. Lett. 78 (1997) 3598 